UCD-96-31

LBNL-39474

October, 1996

\title{
Determining the existence and nature of the quark-gluon plasma by Upsilon suppression at the $\mathrm{LHC}$
}

\author{
J.F. GUnion ${ }^{\dagger}$ \\ Davis Institute for High Energy Physics, Physics Department, \\ University of California, Davis, California 95616 USA \\ R. $\operatorname{VOGT}^{\star}$ \\ Nuclear Science Division, \\ Lawrence Berkeley National Laboratory, Berkeley, California 94720 \\ and \\ Physics Department, \\ University of California, Davis, California 95616 USA
}

\begin{abstract}
Minijet production in $\sqrt{s}=5.5 \mathrm{TeV} \mathrm{Pb}+\mathrm{Pb}$ collisions at the LHC is expected to produce a gluon-dominated plasma with large initial temperatures and energy densities. We discuss the implications of the high initial temperatures on the screening mass and on the relative suppression of members of the $\Upsilon$ family. We demonstrate that $p_{T}$-dependence of the $\Upsilon^{\prime} / \Upsilon$ ratio is only expected if a quark-gluon plasma with significant energy density is present, and that the precise dependence of this ratio will strongly constrain the QGP models. We also propose that the $p_{T}$ spectra of the $\Upsilon$ resonances themselves will provide valuable information by comparing these spectra to that of the $Z$ boson, which should not be influenced by plasma production.

\footnotetext{
${ }^{0 \dagger}$ Supported by the Department of Energy under Contract No. DE-FG03-91ER40674 and by the Davis Institute for High Energy Physics.

${ }^{0 \star}$ This work was supported in part by the Director, Office of Energy Research, Division of Nuclear Physics of the Office of High Energy and Nuclear Physics of the U. S. Department of Energy under Contract No. DE-AC03-76SF0098.
} 


\section{Introduction}

Finite temperature simulations of lattice gauge theory suggest a phase transition at high energy density and temperature to a new phase of QCD matter - the quark-gluon plasma (QGP). In many models, the temperatures and energy densities reached in central nucleus-nucleus collisions at the Brookhaven Relativistic Heavy Ion Collider (RHIC) and the Large Hadron Collider (LHC) at CERN are predicted to be sufficient for QGP formation. Demonstrating that such a QGP has formed and understanding its nature will be a very important component of the experimental programs at both machines. In this paper, we focus on using measurements of the $p_{T}$ spectra of the Upsilon resonance family to accomplish these tasks, emphasizing the possible advantages of the higher LHC energy as compared to RHIC energy.

The details of the QCD phase transition to the QGP, as obtained in finite temperature simulations of lattice gauge theory, are strongly dependent on the number of quark flavors, $n_{f}$, and the number of colors, $N$. The critical temperature, $T_{c}$, and the order of the phase transition are particularly sensitive to the values of $n_{f}$ and $N$ used in the simulations [1]. In a pure $\mathrm{SU}(N)$ gauge theory, $n_{f}=0$, the phase transition is second order, i.e. continuous, for $\mathrm{SU}(2)$ and first order for $\mathrm{SU}(3)$. For the $\mathrm{SU}(N)$ theory, the critical temperature, $T_{c}$, is $260 \mathrm{MeV}$. When dynamical light quarks are included in the lattice simulation, the critical temperature is substantially lower and the order of the phase transition can be altered: for $n_{f}=2, T_{c} \approx 150 \mathrm{MeV}$ and the phase transition appears to be continuous; for simulations with more light flavors, $n_{f} \geq 3, T_{c}$ is unaltered but the transition again becomes first order. This latter conclusion depends on the relative quark masses [2]. The critical energy density at $T_{c}$ is only weakly dependent on the color and flavor degrees of freedom; $\epsilon_{c} \approx 1-2 \mathrm{GeV} / \mathrm{fm}^{3}$ is obtained both with and without quark degrees of freedom for $N=2$ or 3 . For a QGP to be formed in an ultrarelativistic heavy-ion collision, the initial temperature, $T_{0}$, and energy density, $\epsilon\left(t_{0}\right)$, of the quark-gluon system created by the collision must be larger than $T_{c}$ and $\epsilon_{c}$.

Part of the LHC experimental program will be devoted to heavy ion collisions such as $\mathrm{Pb}+\mathrm{Pb}$ at $\sqrt{s}=5.5 \mathrm{TeV}$. At this high energy, the highest available for heavy-ion collisions, perturbative QCD processes will be the dominant factor in determining the initial state of the post-collision quark/gluon system. In particular, at early times, $t_{0} \sim 1 / p_{T} \leq 1 / p_{0}$, semihard production of minijets could be dominant in establishing the initial conditions for further evolution of the system [5]. The appropriate value of $p_{0}$ is uncertain but should be of order $p_{0} \sim 2 \mathrm{GeV}$, in which case $t_{0} \sim 0.1 \mathrm{fm}$. The crucial

\footnotetext{
${ }^{1}$ Minijets are jets with $p_{T} \geq p_{0} \sim 1-3 \mathrm{GeV}$ [3], usually not observable as individual jets below $p_{T} \sim 5 \mathrm{GeV}$ 田.
} 
quantity in determining whether a quark-gluon plasma forms is the initial energy density, given by

$$
\epsilon\left(t_{0}\right)=\frac{\bar{E}_{T} p_{0}}{\pi R^{2}}, \text { with } \bar{E}_{T}=T_{\mathrm{AA}}(\mathbf{0}) \sigma_{\mathrm{jet}}\left\langle E_{T}\right\rangle .
$$

Here, $R$ is of order the nuclear radius, $R_{A}, T_{\mathrm{AA}}(\mathbf{0})$ is the nuclear overlap for a central collision $\left[\right.$ and $\sigma_{\text {jet }}\left\langle E_{T}\right\rangle$ is the first $p_{T}$ moment of the minijet production cross section in the central unity of rapidity in $p p$ collisions (if we neglect nuclear shadowing) using the assumed value of $p_{0}$. The initial temperature $T_{0}$ is proportional to $\left[\epsilon\left(t_{0}\right)\right]^{1 / 4}$. For given $p_{0}, \sigma_{\text {jet }}\left\langle E_{T}\right\rangle$ is computable from QCD once the parton distribution functions (PDF's) for the colliding protons and the types of jets participating in the plasma are both specified. If we take $p_{0}=2 \mathrm{GeV}$, employ MRS D-' [6] parton densities and include only gluon jets (i.e. assume a pure gluon plasma) with rapidity $|y| \leq 0.5$, then the semihard production of minijets leads to a large initial energy density, $\epsilon\left(t_{0}\right)=1170 \mathrm{GeV} / \mathrm{fm}^{3}$, and initial temperature, $T_{0}=1.14 \mathrm{GeV}$, in central $\mathrm{Pb}+\mathrm{Pb}$ collisions at $\sqrt{s}=5.5 \mathrm{TeV}$, to be compared to $\epsilon\left(t_{0}\right)=27 \mathrm{GeV} / \mathrm{fm}^{3}$ and $T_{0}=0.46 \mathrm{GeV}$ at the lower RHIC energy. See [7, 8] for details.

The sensitivity of the critical parameters to the choice of PDF set is illustrated in Table 1, following [7, 8]. MRS D-' provides the most optimistic initial temperature, with the MRS H distributions [9] (designed to fit early HERA data) not far behind. The Duke-Owens-I (DO1) [10, and MRS D0' [6] distributions that grow more slowly as $x$ decreases yield smaller initial energy density. More recent HERA data continues to favor distributions (such as the MRS A parameterization [11]) that at small $x$ are similar to the $\mathrm{H}$ parameterization, i.e. that do not grow quite so rapidly at small- $x$ as the $\mathrm{D}-^{\prime}$ distributions but are certainly much more singular than either DO1 or D0'. All the numbers quoted in the table will become smaller when reductions in parton density due to shadowing in the nucleus are incorporated.

The sensitivity of the results to $p_{0}$ depends upon the PDFs. Increasing $p_{0}$ from 2 to $4 \mathrm{GeV}$ decreases $\sigma_{\text {jet }}\left\langle E_{T}\right\rangle$ by factors of $5,3,2$, and 1.5 for D-' [6], $\mathrm{H}$ [9], D0' [6], and DO1 [10] distributions, respectively, at $\sqrt{s}=5.5 \mathrm{TeV}$. However, $\epsilon\left(t_{0}\right)$, eq. (四), also proportional to $p_{0}$, changes by a factor of two or less, even increasing in the DO1 case. The sensitivity of $\epsilon\left(t_{0}\right)$ to $p_{0}$ at RHIC is much greater, declining by as much as a factor of 5 between $p_{0}=2 \mathrm{GeV}$ and $p_{0}=4 \mathrm{GeV}$. Inclusion of quark as well as gluon jets would increase $\epsilon\left(t_{0}\right)$ by roughly $30 \%$ at $\sqrt{s}=5.5 \mathrm{TeV}$, but if the plasma is a quark-gluon plasma with 2 to 3 active flavors, the equation of state changes so that $T_{0}$ actually declines.

\footnotetext{
${ }^{2}$ The nuclear overlap function for a collision of projectile $A$ with target $B$ is $T_{A B}(\mathbf{b})=$ $\int d^{2} \mathbf{s} d z d z^{\prime} \rho_{A}(\mathbf{s}, z) \rho_{B}\left(\mathbf{b}-\mathbf{s}, z^{\prime}\right)$ where the impact parameter $\mathbf{b}$ is zero in the most central collisions.
} 


\begin{tabular}{|c|c|c|c|c|c|}
\hline PDF & \#jets & \# gluon jets & $\bar{E}_{T}(\mathrm{GeV})$ & $T_{0}(\mathrm{GeV})$ & $d N_{\text {ch }}^{\text {gluons only }} / d y$ \\
\hline DO1 & 776 & 640 & 3200 & 0.74 & 914 \\
\hline D0 & 1510 & 1250 & 5440 & 0.81 & 1350 \\
\hline H & 3250 & 2710 & 10300 & 0.96 & 2130 \\
\hline D- $^{\prime}$ & 5980 & 5220 & 17600 & 1.14 & 3360 \\
\hline
\end{tabular}

Table 1: Dependence of initial temperature and particle density on distribution function choice at $\sqrt{s}=5.5 \mathrm{TeV}$. The results are taken from Ref. [8]. We employ $T_{\mathrm{PbPb}}(\mathbf{0})=32 \mathrm{mb}^{-1}$. The distributions employed are Duke-Owens I, DO1 [10], the MRS D0 ${ }^{\prime}$ and $\mathrm{D}-^{\prime}$ from [G], and the MRS $\mathrm{H}$ distributions from 9 .

Regardless of the parameters employed, it is apparent that minijet production yields substantially larger initial temperatures than previously predicted (for example, in Ref. [12]) and thus enhances the possibility of QGP production in thermal equilibrium. Only the extent of this enhancement is uncertain. For large enough $p_{0}$ and/or extensive nuclear shadowing, the energy density and temperature might be reduced sufficiently that the plasma would not be fully equilibrated, although a QGP might still be formed. In any case, it is important to examine the proposed signatures of plasma formation for the increased initial energy density and temperature obtained after including the effects of minijet production.

One of the proposed signatures of the QCD phase transition is the suppression of quarkonium production, particularly of the $J / \psi$ [13, 14]. In fact, $J / \psi$ and $\psi^{\prime}$ suppression have been observed in nucleus-nucleus collisions at the CERN SPS with $\sqrt{s}=19.4 \mathrm{GeV}$ [15, 16]. In a QGP, the suppression occurs due to the shielding of the $c \bar{c}$ binding potential by color screening, leading to the breakup of the resonance. The $c \bar{c}\left(J / \psi, \psi^{\prime}, \cdots\right)$ and $b \bar{b}(\Upsilon$, $\left.\Upsilon^{\prime}, \ldots\right)$ resonances have smaller radii than light-quark hadrons and therefore higher temperatures are needed to disassociate these quarkonium states. At current energies, the situation for the $J / \psi$ is rather ambiguous because the bound state can also break up through interactions with nucleons and comoving hadrons - QGP production is not a unique explanation of $J / \psi$ suppression [17, 18, 19, 20]. At LHC energies there will continue to be ambiguity in this regard, and, in addition, $J / \psi$ and $\psi^{\prime}$ detection efficiencies may not be large [21].

In contrast, the $\Upsilon$ resonances are sufficiently tightly bound that it is more difficult to break them up as a consequence of ordinary multiple interactions. In addition, they can be detected in the $\mu^{+} \mu^{-}$decay mode with relatively high efficiency at the LHC (e.g. $33 \%$ vs. $6 \%$ for $J / \psi$ in the CMS detector 
21]). Further, this detection efficiency derives entirely from the central region $(|\eta|<2.4$ in the CMS detector [21]), where the predictions for the relative suppression of the members of the $\Upsilon$ family in the presence of a gluon plasma can be most reliably tested (given that the baryon density is predicted to be low and should not play a significant role). However, it was previously assumed that the $\Upsilon$ would not be suppressed by QGP production [14, 22], since the $\Upsilon$ is much smaller than the $c \bar{c}$ and other $b \bar{b}$ resonances (such as the $\Upsilon^{\prime}$ and $\chi_{b}$ states), implying that a much higher temperature than expected from earlier estimates of $T_{0}$ is needed to dissociate the $\Upsilon$ [14]. In view of the high initial temperature of a gluon plasma, $T_{0} \approx 1 \mathrm{GeV}$, when minijets are included, a reexamination of this assumption is in order. If $\Upsilon$ suppression by a minijet plasma is predicted, the $\Upsilon$ may provide a cleaner plasma signature than the $J / \psi$ because of its weaker interactions with matter. In high energy heavy-ion collisions, the $\Upsilon$ rate should be large enough to test the predictions given here and help clarify the initial state of the system.

The paper is organized as follows. In the next section, we review the model of quarkonium suppression in a QGP and introduce two different scenarios for quarkonium breakup at finite temperature. Section 3 presents the quarkonium production model we adopt and includes a discussion of the expected $\Upsilon$ production rate. We then provide a detailed examination of $\Upsilon$ family suppression in a minijet plasma as a function of $p_{T}$ and discuss the sensitivity of the results to the initial conditions. In section 5 , we elucidate the steps necessary to extract information on the state of the system. We particularly focus on the $\Upsilon^{\prime} / \Upsilon$ ratio and discuss the effect of cascade decays from higher states on the signal. We also propose using the $Z$ boson as a reference process against which $\Upsilon$ suppression can be tested. In summary, we show that the $\Upsilon$ family may provide an extremely powerful tool for diagnosing the existence and nature of the quark-gluon plasma.

\section{Quarkonia in a quark-gluon plasma}

We follow the arguments of Ref. [14] to discuss the finite temperature properties of quarkonium. The interquark potential for nonrelativistically bound quarkonium at zero temperature is

$$
V(r, 0)=\sigma r-\frac{\alpha}{r},
$$

where $r$ is the separation between the $Q$ and $\bar{Q}$. The parameters are $\sigma=0.192$ $\mathrm{GeV}^{2}, \alpha=0.471, m_{c}=1.32 \mathrm{GeV}$ and $m_{b}=4.746 \mathrm{GeV}$ 14. This potential is modified at finite temperatures due to color screening, becoming

$$
V(r, T)=\frac{\sigma}{\mu(T)}\left(1-e^{-\mu(T) r}\right)-\frac{\alpha}{r} e^{-\mu(T) r} .
$$


The screening mass, $\mu(T)$, is an increasing function of temperature. When $\mu(T=0) \rightarrow 0$, eq. (2) is recovered. At finite temperature, when $r \rightarrow 0$, the $1 / r$ behavior is dominant while as $r \rightarrow \infty$ one finds that the range of the potential decreases with $\mu(T)$, making the binding less effective at finite temperature. Semiclassically the energy is

$$
E(r, T)=2 m_{Q}+\frac{c}{m_{Q} r^{2}}+V(r, T),
$$

where $\left\langle p^{2}\right\rangle\left\langle r^{2}\right\rangle=c \approx \mathcal{O}(1)$ [14]. Minimizing $E(r, T)$ gives the radius of the bound state at each $T$. For $\mu(T)$ above a certain critical value, $\mu_{D}$, there is no longer a minimum and the screening has become strong enough for binding to be impossible and the resonance no longer forms in the plasma. The temperature $T_{D}$ at which this happens is that for which $\mu\left(T_{D}\right)=\mu_{D}$.

The values of $\mu_{D}$ for the bottonium states are given in Table 2, along with the bottonium mass at $\mu=\mu_{D}, M\left(\mu_{D}\right)$. Also shown are the results for the charmonium states. We note that $\mu_{D}$ and $M\left(\mu_{D}\right)$ are independent of $T_{D}$, but that $T_{D}$ depends upon the functional form of $\mu(T)$. Table 2 shows that the $\Upsilon$ mass is actually larger at breakup than at $T=0$ because the $1 / r$ contribution to the potential is dominant, whereas the $\Upsilon^{\prime}$ and $\chi_{b}(1 P)$ masses are smaller due to the fact that the linear term is more important for these resonances.

\begin{tabular}{|c|c|c|c|c|c|c|}
\hline & $\Upsilon$ & $\Upsilon^{\prime}$ & $\chi_{b}(1 P)$ & $J / \psi$ & $\psi^{\prime}$ & $\chi_{c}(1 P)$ \\
\hline$M(\mathrm{GeV})$ & 9.445 & 10.004 & 9.897 & 3.07 & 3.698 & 3.5 \\
\hline$r(\mathrm{fm})$ & 0.226 & 0.509 & 0.408 & 0.453 & 0.875 & 0.696 \\
\hline$\tau_{F}(\mathrm{fm})$ & 0.76 & 1.9 & 2.6 & 0.89 & 1.5 & 2.0 \\
\hline$M\left(\mu_{D}\right)(\mathrm{GeV})$ & 9.615 & 9.778 & 9.829 & 2.915 & 3.177 & 3.198 \\
\hline$\mu_{D}(\mathrm{GeV})$ & 1.565 & 0.671 & 0.558 & 0.699 & 0.357 & 0.342 \\
\hline
\end{tabular}

Table 2: Properties of the quarkonium states both at zero temperature and at $T=T_{D}$ (the breakup temperature for each bound state), taken from Ref. [14]. The masses at $T=0$ are from the solution to the Schrodinger equation with the potential of eq. (2). Note that $r$ is the distance between the $Q$ and $\bar{Q}$ and $\tau_{F}$ is the formation time of the bound state. $M\left(\mu_{D}\right)$ and $\mu_{D}$ are the mass at breakup and the screening mass at $T_{D}$; both are independent of the actual value of $T_{D}$ as determined by the functional form of $\mu(T)$.

It is necessary to know the dependence of the screening mass on temperature before the breakup temperature $T_{D}$ can be determined. Since the behavior of $\mu(T)$ is unknown for $T \gg T_{c}$, we consider two scenarios which may be expected to bracket the realistic situation. 
- The first is a parameterization based on $\mathrm{SU}(N)$ lattice simulations [23],

$$
\frac{\mu(T)}{T_{c}} \simeq 4 \frac{T}{T_{c}}
$$

which results in the lowest values of $T_{D}$. Although this parameterization of $\mu(T)$ is independent of $T_{c}$, we will present our results as a function of the ratio $T / T_{c}$ taking $T_{c}=260 \mathrm{MeV}$, consistent with $\mathrm{SU}(3)$ lattice calculations. For this value of $T_{c}$, the $\Upsilon$ breaks up at $T_{D} / T_{c} \approx 1.5$. In Ref. [22], this parameterization was used with $T_{c}=150$ $\mathrm{MeV}$, leading to the estimate $T_{D} / T_{c} \sim 2.6$ for the $\Upsilon$.

- The second is an estimate from perturbation theory [24],

$$
\frac{\mu(T)}{T_{c}}=\sqrt{1+\frac{n_{f}}{6}} g\left(\frac{T}{T_{c}}\right) \frac{T}{T_{c}},
$$

where the temperature-dependent running coupling constant is

$$
g^{2}\left(\frac{T}{T_{c}}\right)=\frac{24 \pi^{2}}{\left(33-2 n_{f}\right) \ln \left[\left(19 T_{c} / \Lambda_{\overline{\mathrm{MS}}}\right)\left(T / T_{c}\right)\right]} .
$$

In $\mathrm{SU}(3)$ gauge theory, $T_{c} / \Lambda_{\overline{\mathrm{MS}}}=1.78 \pm 0.03$ [14]. In this case, $T_{D}$ can be quite high and depends strongly on $n_{f}$ and $T_{c}$. We present results using eq. (6) with $n_{f}=3$ and $T_{c}=150 \mathrm{MeV}$.

We shall refer to these two distinct choices for $\mu(T)$ as the $\mathrm{SU}(N)$ and 3Flavor QGP's, respectively. The temperature dependence for both estimates of $\mu(T)$ is shown in Fig. 1 with $\mu_{D}$ indicated for $\Upsilon, \Upsilon^{\prime}$ and $\chi_{b}$. The values of $T_{D}$ are given in Table 3 for both cases. Even in the first case, the early estimates of initial temperatures of order $T_{0} \sim 260 \mathrm{MeV}$ for the LHC implied that $\Upsilon$ suppression would be unlikely [12], and that even $\Upsilon^{\prime}$ and $\chi_{b}$ suppression would not be certain.

In contrast, the higher 'minijet' value of $T_{0} \sim 1.14 \mathrm{GeV}$, combined with the results of Fig. 11 and the $T_{D}$ values given in Table 3 indicate that, when the $\mathrm{SU}(N)$ parameterization is used, the $\Upsilon$ may easily be suppressed in the minijet gluon plasma at LHC energies. However, the 3-Flavor estimate leads to much higher breakup temperatures for the Upsilon states and the distinct possibility that the $\Upsilon$ will not be suppressed ${ }^{[}$even though the $\Upsilon^{\prime}$ and $\chi_{b}$ could be suppressed. In either case, the suppression pattern depends on the space-time evolution of the plasma. Depending upon circumstances, this dependence could be either an impediment to the extraction of the physics of

\footnotetext{
${ }^{3}$ Note that $\Upsilon$ production from $\Upsilon^{\prime}$ and $\chi_{b}$ decays will be suppressed if the $\Upsilon^{\prime}$ and $\chi_{b}$ states are suppressed.
} 


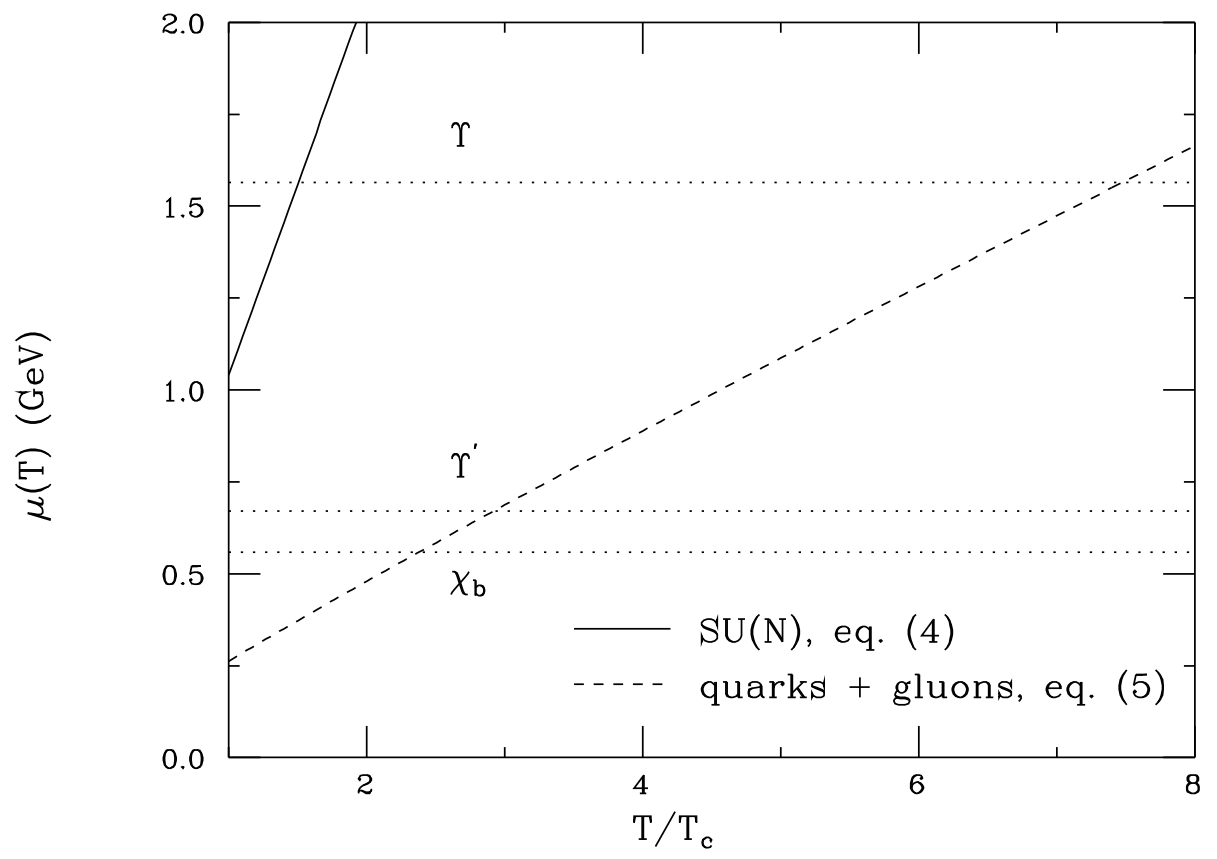

Figure 1: The screening mass as a function of temperature for the scenarios of eqs. (5) (solid) and (6) (dashed). The values of $\mu_{D}$ at breakup for the $\Upsilon, \Upsilon^{\prime}$, and $\chi_{b}$ are also indicated by the dotted lines.

the QGP or a very powerful tool. In most scenarios, much can be learned about the QGP by simply examining the ratio [22] of production rates for higher $b \bar{b}$ resonances to the production rate for the $\Upsilon$. However, for a quantitative determination of QGP suppression on an absolute scale, a benchmark such as $Z$ production, to which $\Upsilon$ production cross sections may be compared, is needed.

\section{Cross sections, rates, and the color evapo- ration model}

Before turning to the details of $\Upsilon$ suppression, let us determine whether or not the $\Upsilon$ production rate is large enough for any suppression to be observable. An estimate of the $\Upsilon$ yield is not straightforward since the formation of bound states from the predominantly color octet $Q \bar{Q}$ pairs is nonperturbative and therefore model-dependent.

The color evaporation model [25] has often been applied to the production of quarkonium states below the open charm/bottom thresholds. The $Q \bar{Q}$ pair neutralizes its color by interaction with the collision-induced color field- 


\begin{tabular}{|c|c|c|}
\hline \multicolumn{3}{|c|}{$T_{D}(\mathrm{MeV})$} \\
\hline & $\mathrm{SU}(N)$ only, eq. (5) & gluons and quarks, eq. (6) \\
\hline$\Upsilon$ & 391 & 1125 \\
\hline$\Upsilon^{\prime}$ & 260 & 447 \\
\hline$\chi_{b}$ & 260 & 368 \\
\hline$J / \psi$ & 260 & 459 \\
\hline$\psi^{\prime}$ & 260 & 211.5 \\
\hline$\chi_{c}$ & 260 & 204 \\
\hline
\end{tabular}

Table 3: The values of $T_{D}$ (in $\mathrm{MeV}$ ) for the two choices of $\mu(T)$, eq. (5) and eq. (6). We employ $T_{c}=260 \mathrm{MeV}$ for the $\mathrm{SU}(N)$ case and $T_{c}=150 \mathrm{MeV}$ in the case of eq. (6). The lower $T_{c}$ used in the 3-Flavor case results in the lower $T_{D}$ values for the $\psi^{\prime}$ and $\chi_{c}$.

"color evaporation". The $Q$ and the $\bar{Q}$ either combine with light quarks to produce heavy-flavored hadrons or bind with each other in a quarkonium state. The additional energy needed to produce heavy-flavored hadrons is obtained nonperturbatively from the color field in the interaction region. Depending on $m_{Q}$, the yield of all quarkonium states may be only a small fraction of the total $Q \bar{Q}$ cross section below the $\sqrt{\hat{s}}=2 m_{H}$ threshold, where $m_{H}$ is the mass of the lightest heavy-flavored meson. The total heavy quark cross section below this $H \bar{H}$ threshold, $\tilde{\sigma}$, is calculated at leading order by integrating over the $Q \bar{Q}$ pair mass from $2 m_{Q}$ to $2 m_{H}$,

$$
\tilde{\sigma}(s)=\sum_{i, j} \int_{4 m_{Q}^{2}}^{4 m_{H}^{2}} d \hat{s} \int d x_{1} d x_{2} f_{i / p}\left(x_{1}\right) f_{j / p}\left(x_{2}\right) \sigma_{i j}(\hat{s}) \delta\left(\hat{s}-x_{1} x_{2} s\right)
$$

where $i j=q \bar{q}$ or $g g$ and $\sigma_{i j}(\hat{s})$ is the $i j \rightarrow Q \bar{Q}$ subprocess cross section. The color evaporation model was taken to next-to-leading order (NLO) using the exclusive $Q \bar{Q}$ hadroproduction calculation [26] to obtain the energy, $x_{F}$, and $p_{T^{-}}$-dependence of quarkonium production [27, 28].

The division of $\tilde{\sigma}$ into heavy flavored hadrons vs. quarkonium and the relative quarkonium production rates are both a matter of assumption in the color evaporation model. For the model to have any predictive power, the relative quarkonium production rates should be independent of projectile, target, and energy. Experiment suggests that this is true for the charmonium ratios $\chi_{c} / \psi$ and $\psi^{\prime} / \psi$ over a broad energy range [29, 30, 31, 32]. The available bottonium data follows this trend: $\Upsilon^{\prime} / \Upsilon=0.53 \pm 0.13$ and $\Upsilon^{\prime \prime} / \Upsilon=0.17 \pm$ 0.06 for proton beams at 400 [33] and $800 \mathrm{GeV}$ [34, 35]. These results are consistent with the production of $\Upsilon$ states in $p \bar{p}$ collisions at the Tevatron [36]. The color evaporation model also reproduces the energy dependence 
of charmonium and bottonium production over a wide range of energy as well as most of the $x_{F}$ dependence of the charmonium states ${ }^{\boxplus}$, provided the fraction of quarkonium vs. $H \bar{H}$ states is assumed to be independent of all kinematic variables.

With these assumptions and our knowledge of the $\Upsilon^{\prime} / \Upsilon$ and $\Upsilon^{\prime \prime} / \Upsilon$ ratios at low energy, the normalization of each quarkonium state can be fixed empirically from data, allowing predictions of the production cross sections at LHC energies. The procedure we have followed is described below.

- As a first step, we assess the accuracy of the model for describing existing $p p / p \bar{p}$ data. Fixed target $\Upsilon$ data has been generally given as the sum of $\Upsilon, \Upsilon^{\prime}$, and $\Upsilon^{\prime \prime}$ production. From this data, one can determine $B(d \sigma / d y)_{y=0}$ where $B$ is an effective dilepton branching ratio reflecting the production yield through the $\Upsilon, \Upsilon^{\prime}$, and $\Upsilon^{\prime \prime}$ resonances. As shown in Fig. 2, taken from Ref. [27], a good fit to the data, up to and including ISR energies [33, 34, 35, 38, 39], is obtained with

$$
B\left(\frac{d \sigma(s)}{d y}\right)_{y=0}=1.6 \times 10^{-3}\left(\frac{d \tilde{\sigma}(s)}{d y}\right)_{y=0},
$$

where $d \tilde{\sigma} / d y$ is computed using the MRS D-' [6] or GRV HO [40] PDFs, taking $m_{b}=4.75 \mathrm{GeV}$ and the renormalization and factorization scales set to $\mu=m_{T, b \bar{b}}=\sqrt{m_{b}^{2}+\left(p_{T, b}^{2}+p_{T, \bar{b}}^{2}\right) / 2}$. The high energy data from UA1 [41] and CDF [36] are also shown in Fig. 2 and agree very well with the energy dependence of the color evaporation model, as obtained from eq. (8). Both sets of PDFs give compatible predictions at the LHC energy. The predicted cross section is up to a factor of 20 larger at $5.5 \mathrm{TeV}$ than that given by the empirical parameterization, $d \sigma /\left.d y\right|_{y=0} \propto \exp \left(-14.7 M_{\Upsilon} / \sqrt{s}\right)$ [42, 43], labeled CR in Fig. 2.

- The second step is to extract the $\Upsilon, \Upsilon^{\prime}$ and $\Upsilon^{\prime \prime}$ components separately using the $\Upsilon^{\prime} / \Upsilon$ and $\Upsilon^{\prime \prime} / \Upsilon$ ratios quoted earlier, and the known branching ratios $B \Upsilon_{i} \equiv B\left(\Upsilon_{i} \rightarrow \mu^{+} \mu^{-}\right)$where $\Upsilon_{i}$ represents the individual $\Upsilon$ states. If we write $d \sigma_{\Upsilon_{i}} /\left.d y\right|_{y=0} \equiv f_{\Upsilon_{i}} d \tilde{\sigma} /\left.d y\right|_{y=0}$ for the $\Upsilon_{i}$ cross sections, then from eq. (9)

$$
f_{\Upsilon} B_{\Upsilon}+f_{\Upsilon^{\prime}} B_{\Upsilon^{\prime}}+f_{\Upsilon^{\prime \prime}} B_{\Upsilon^{\prime \prime}}=B=0.0016 .
$$

Using $f_{\Upsilon^{\prime}} / f_{\Upsilon}=0.53$ and $f_{\Upsilon^{\prime \prime}} / f_{\Upsilon}=0.17$, as quoted earlier, and $B_{\Upsilon_{i}}$ from the Particle Data Book [44], we find [28]

$$
f_{\Upsilon}=0.044, \quad f_{\Upsilon^{\prime}}=0.023, \quad f_{\Upsilon^{\prime \prime}}=0.0074 .
$$

\footnotetext{
${ }^{4}$ At high $x_{F}$, additional production from other mechanisms such as intrinsic heavy quarks [37] may be important.
} 


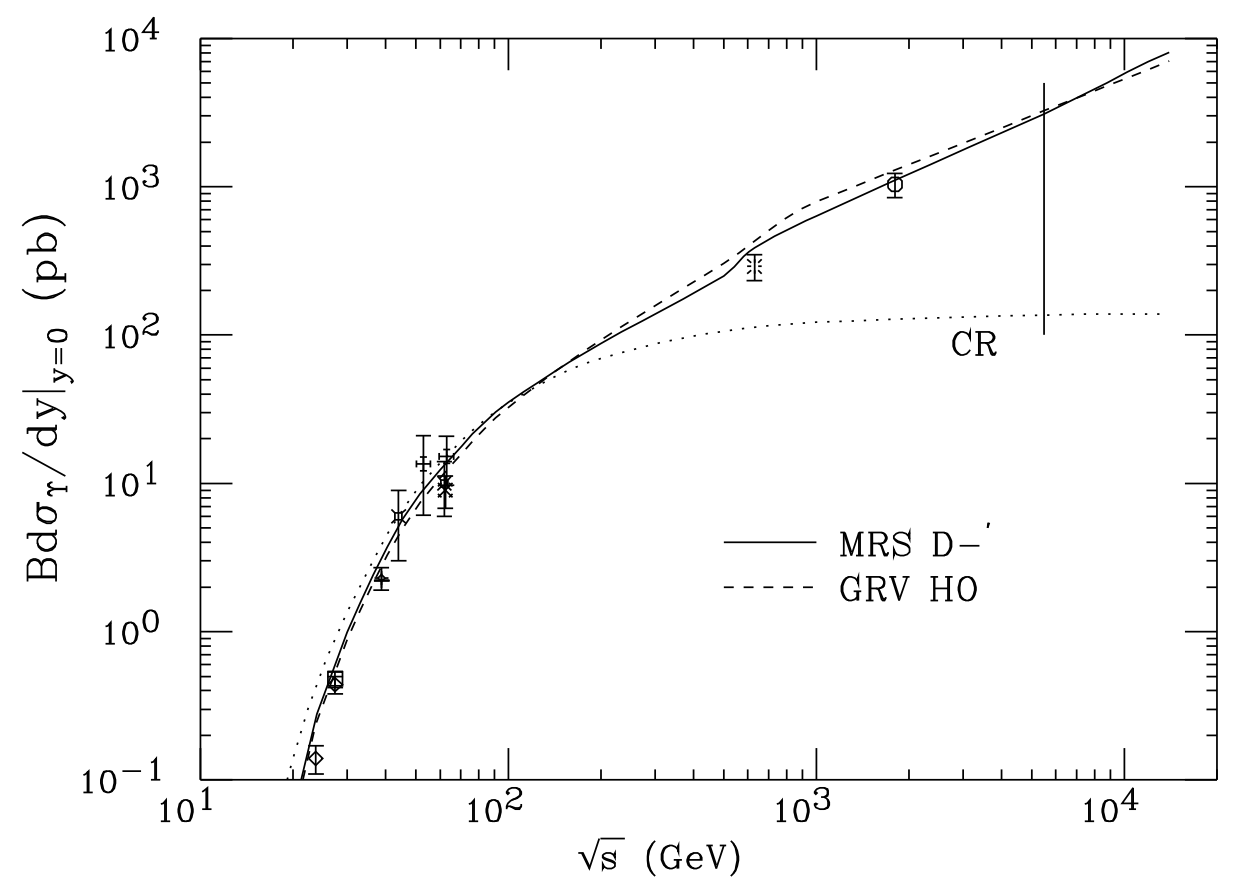

Figure 2: We show $B d \sigma /\left.d y\right|_{y=0}$ for $\Upsilon+\Upsilon^{\prime}+\Upsilon^{\prime \prime}$ in $p p$ collisions, as indicated in eq. (9), for the MRS D-' [6] (solid) and GRV HO [40] (dashed) parton densities. Also shown is the empirical Craigie parameterization [42, 43] (dotted). The data are taken from [33, 34, 36, 38, 39, 41]. The vertical solid line indicates the maximum LHC $\mathrm{Pb}+\mathrm{Pb}$ energy. This figure was first presented in Ref. [27].

- Thirdly, we must separate direct from indirect production. The measured $\Upsilon_{i}$ production cross sections, or equivalently the $f_{\Upsilon_{i}}$, are only effective values which reflect both direct production and chain decays of higher mass states. Isolation of the direct production cross sections for each $\Upsilon_{i}$ requires detection of the radiated photons associated with chain decays. Data which allows this separation is not currently available. As discussed later, the extent to which the required photon detection will be possible at the LHC is also uncertain. We shall see that this is a very important issue. For now, we make the assumptions:

$$
f_{\chi_{b i}(1 P)}^{d}=f_{\Upsilon^{\prime}} ; \quad f_{\chi_{b i}(2 P)}^{d}=f_{\Upsilon^{\prime \prime}}
$$

for each $i$, where $i=0,1,2$ labels the $\chi_{b}$ states in PDG notation [44], and the $d$ superscript indicates the $f$ for direct production. With this and the summed branching ratios [4]

$$
\sum_{i=0,1,2} B\left(\chi_{b i}(1 P) \rightarrow \Upsilon \gamma\right) \sim 0.63
$$




$$
\begin{aligned}
& \sum_{i=0,1,2} B\left(\chi_{b i}(2 P) \rightarrow \Upsilon \gamma\right) \sim 0.16 \\
& \sum_{i=0,1,2} B\left(\chi_{b i}(2 P) \rightarrow \Upsilon^{\prime} \gamma\right) \sim 0.42
\end{aligned}
$$

along with $B\left(\Upsilon^{\prime} \rightarrow \Upsilon X\right) \sim 0.27$ and $\Upsilon^{\prime \prime}$ decays to $\Upsilon^{\prime}, \Upsilon$ and $\chi_{b i}(2 P)$, we can compute the $f^{d}$ 's for direct production of all the $\Upsilon_{i}$. One finds that about 0.014 of the total $f_{\Upsilon}=0.044$ is due to $\chi_{b i}(1 P)$ decays. This would be similar to what is found in the analogous $\psi, \chi_{c}$ situation. Similarly, $\sim 0.001$ of $f_{\Upsilon}$ and $\sim 0.003$ of $f_{\Upsilon^{\prime}}$ would be due to $\chi_{b i}(2 P)$ decays. Also important are $\Upsilon^{\prime} \rightarrow \Upsilon X$ decays; $f_{\Upsilon^{\prime}}$ [eq. (11)] implies that an additional 0.006 of $f_{\Upsilon}$ would be indirect. The contributions from chain decays of the $\Upsilon^{\prime \prime}$ are small. Also, there are no contributions to the $\Upsilon^{\prime \prime}$ rate coming from higher states that are known to be significant. Altogether, we would have:

$$
\begin{gathered}
f_{\Upsilon}^{d} \sim 0.023, f_{\Upsilon^{\prime}}^{d} \sim 0.020, f_{\Upsilon^{\prime \prime}}^{d} \sim 0.0074 \\
f_{\chi_{b i}(1 P)}^{d} \sim 0.023, f_{\chi_{b i}(2 P)}^{d} \sim 0.0074,
\end{gathered}
$$

where $i=0,1,2$ labels the different $\chi_{b i}(1 P, 2 P)$ states. Note that only about half of $f_{\Upsilon}$ is due to direct $\Upsilon$ production. The results in eq. (13) imply that about $14 \%$ of $\tilde{\sigma}$ goes into bottonium state production.

In Table 4, we give the corresponding normalized direct production cross sections in $p p$ collisions, $f^{d} \tilde{\sigma}$, for each state with $f^{d}$ from eq. (13) and $\tilde{\sigma}$ computed using the MRS D-' parton densities at 5.5 TeV. The cross sections given in Table 4 are integrated over all rapidity. At this energy, the results with the GRV HO distributions are about $20 \%$ smaller.

We would like to comment on the differences between the color evaporation model used here and other models of quarkonium production. The color singlet model [45], in which bound states with the right quantum numbers are directly produced, has been shown to be insufficient to reproduce the Tevatron data on $J / \psi, \psi^{\prime}$, and $\Upsilon$ production [29, 36]. The low- $p_{T} \Upsilon$ production data from the Tevatron has recently been explained using a model of color octet production involving corrections to the bound state wavefunctions at higher order in the relative velocity of the $Q \bar{Q}$ where $\alpha_{s} \sim v$ for the bound state 46]. We would like to point out that the $p_{T}$-dependence of quarkonium production is quite similar to the $p_{T}$-dependence of unbound $Q \bar{Q}$ pairs [26]. This same dependence also appears in the color octet model since the octet mechanism makes other ${ }^{3} S_{1}$ production channels available. Thus the color octet model is quite similar in spirit to the color evaporation model considered here. However, at larger $p_{T}, p_{T}>m_{\Upsilon}$, fragmentation processes such as $g \rightarrow \Upsilon$ and $b \rightarrow \Upsilon$ 477 should eventually dominate the production processes considered in the color octet model. It has been recently shown that 


\begin{tabular}{|c|c|c|c|c|c|}
\hline & $\Upsilon$ & $\Upsilon^{\prime}$ & $\Upsilon^{\prime \prime}$ & $\chi_{b}(1 P)$ & $\chi_{b}(2 P)$ \\
\hline$f^{d} \tilde{\sigma}(\mathrm{nb})$ & 426 & 233 & 137 & 426 & 137 \\
\hline$N^{d}(\mathrm{PbPb})$ & $2.02 \times 10^{6}$ & $1.75 \times 10^{6}$ & $6.47 \times 10^{5}$ & $2.02 \times 10^{6}$ & $6.47 \times 10^{5}$ \\
\hline$N_{\mu \mu}^{d}(\mathrm{PbPb})$ & $4.99 \times 10^{4}$ & $2.30 \times 10^{4}$ & $1.17 \times 10^{4}$ & - & - \\
\hline
\end{tabular}

Table 4: The normalized cross sections, $f^{d} \tilde{\sigma}$, for directly produced bottonium states in $p p$ collisions at $5.5 \mathrm{TeV}$, using the $f^{d}$ 's of eq. (13) and the prediction of eq. (8) that $\tilde{\sigma}=17.35 \mu \mathrm{b}$ [27], where the MRS D-' parton densities are employed with $m_{b}=4.75 \mathrm{GeV}$ and $\mu=m_{T, b \bar{b}}$. Also given is the number $N^{d}$ of each type of bottonium state produced directly in $\mathrm{Pb}+\mathrm{Pb}$ collisions at the LHC, calculated using eq. (14) with $\sigma_{N N}=60 \mathrm{mb}, T_{\mathrm{PbPb}}(\mathbf{0})=32 \mathrm{mb}^{-1}$ and $L_{\text {int }}^{\mathrm{PbPb}}=2.59 \mathrm{nb}^{-1}$. For $\Upsilon, \Upsilon^{\prime}, \Upsilon^{\prime \prime}$ we give the corresponding number of $\mu^{+} \mu^{-}$ pairs from decay of directly produced states.

the Tevatron charmonium and bottonium $p_{T}$ data is also in good agreement with the color evaporation model at NLO, as described in [28]. In the color evaporation picture, $g g$ scattering followed by the splitting $g \rightarrow b \bar{b}$ incorporated at NLO is similar to models of $g \rightarrow \Upsilon$ fragmentation [47. By including this splitting, the color evaporation model provides a good description of the quarkonium $p_{T}$ distributions.

We can now employ the results for $f^{d}$ given in Table 4 to predict rates for direct production of bottonium states in $\mathrm{Pb}+\mathrm{Pb}$ collisions at the LHC. For central collisions, the expected rates are given by

$$
N^{d}=\sigma_{N N} T_{\mathrm{PbPb}}(\mathbf{0}) f^{d} \tilde{\sigma} L_{\mathrm{int}}^{\mathrm{PbPb}},
$$

where $\sigma_{N N} T_{\mathrm{PbPb}}(\mathbf{0})$ is the number of central $\mathrm{Pb}+\mathrm{Pb}$ collisions f and $f^{d} \tilde{\sigma}$, with $f^{d}$ taken from eq. (13), is the cross section for direct production of a given bottonium state in $p p$ collisions. In one month of running the integrated luminosity for lead beams is expected to be $L_{\mathrm{int}}^{\mathrm{Pb}}=2.59 / \mathrm{nb}$. Typical rates are given in Table 4 ; they are on the order of $10^{6}$ for $\Upsilon$ and $\Upsilon^{\prime}$. Approximately $10-15 \%$ of the cross section is within $|\eta| \leq 1$. The number of muon pairs from the $\Upsilon, \Upsilon^{\prime}$ and $\Upsilon^{\prime \prime}$ decays, found by multiplying the total number of $\Upsilon_{i}$ directly produced, $N^{d}$, by the appropriate branching ratio, is also given in Table 4 . These rates suggest that production and suppression of these states should be measurable by CMS in the very clean $\mu^{+} \mu^{-}$final state decay mode. The rapidity distributions are rather broad and nearly constant out to $y \simeq 4$ with the MRS D-' parton distributions. The GRV HO results exhibit a somewhat narrower rapidity plateau [27.

\footnotetext{
${ }^{5}$ We use $\sigma_{N N} \approx 60 \mathrm{mb}$, assuming that the high energy rise in $\sigma_{p \bar{p}}$ will also hold for $\sigma_{N N}$, and $T_{\mathrm{PbPb}}(\mathbf{0}) \simeq 32 / \mathrm{mb}$.
} 
The rates of Table 4 are those obtained by simply multiplying the $p p$ cross section by the expected number of central $\mathrm{Pb}+\mathrm{Pb}$ collisions. This procedure does not include any nuclear effects (e.g. shadowing or absorption) on $\Upsilon$ production. However, significant nuclear effects have been observed in fixedtarget interactions. The E772 collaboration has measured a less than linear $A$ dependence for $\Upsilon$ production at $\sqrt{s}=38.8 \mathrm{GeV}, \sigma_{p A}^{\Upsilon}=\sigma_{p p}^{\Upsilon} A^{\alpha}$ where $\alpha=0.96$ [48]. If no nuclear effects were present, the production cross section would grow as $A^{1}$. Note that $\alpha=0.96$ implies a $20 \%$ reduction in the $p \mathrm{~Pb}$ production cross section relative to the $A^{1}$ prediction. The two possible sources of this reduction in $p A$ collisions are shadowing and absorption.

- If the reduction is due to shadowing, the observed greater suppression (smaller $\alpha$ ) of $J / \psi$ compared to $\Upsilon$ implies that the shadowing of the gluon PDFs is greater at smaller $x$ values. This would then imply that, for the $\Upsilon$ (or any other given onium resonance), shadowing would be greater at higher energies due to the lower $x$ region probed. In heavyion collisions at LHC energies the shadowing mechanism could reduce $\sigma_{\mathrm{PbPb}}^{\Upsilon}$ by $25-40 \%$ relative to the result obtained assuming proportionality to $A^{2}$ 49]. The larger shadowing effect expected for $\mathrm{Pb}+\mathrm{Pb}$ collisions compared to $\mathrm{pPb}$ collisions comes from the convolution of two nuclei with modified parton densities. To the extent that shadowing in central collisions is the same as for minimum bias collisions (which include peripheral as well as central collisions), the same $25-40 \%$ reduction should be applied to the numbers of Table 4 . However, nucleons near the nuclear surface might experience a smaller shadowing effect than those in the center of the nucleus.

- On the other hand, the deviation of the $A$ dependence from unity in fixed-target interactions has also been attributed to absorption by nucleons; interactions with comoving secondaries are possible although rare in $p A$ collisions. In this approach, $\alpha$ is related to the the effective onium absorption cross section in nuclear matter by $1-\alpha \approx$ $9 \sigma_{\text {abs }} / 16 \pi r_{0}^{2}$, assuming a step function nuclear shape. The apparent $\Upsilon$ absorption cross section is 40-50\% less than the $\psi$ absorption cross section, suggesting that the $\Upsilon$ suffers fewer final-state interactions than the $J / \psi$ (possibly because of its smaller size). The onium-nucleon absorption cross section has been calculated on the basis of the operatorproduct expansion [50, 51] and has been shown to quickly attain its asymptotic value [52. Absorption by nucleons has been shown to be less effective in peripheral relative to central nuclear collisions, see e.g. [18, 20]. Therefore nucleon interactions with the $J / \psi$ may be insufficient to account for $J / \psi$ measurements in heavy-ion interactions at current energies [15, 16]. Secondary particle production increases in 
central relative to peripheral collisions, increasing the influence of comovers in central collisions as well as in nuclear collisions compared to $p A$ interactions [20]. The onium-comover cross section can be estimated from the onium-nucleon absorption cross section by the additive quark model assuming the comovers are mesons [53].

A mixture of shadowing and absorption is most likely. If the value of $\alpha$ depends on the Upsilon state, absorption is important: $\sigma_{\text {abs }}$ could increase with the size of the Upsilon state [54]. If shadowing were dominant, $\alpha$ would remain essentially unchanged since the $x$ values probed by the different Upsilon states are very nearly equal. In fact, the E772 collaboration observed no difference in the $J / \psi$ and $\psi^{\prime} A$ dependence or in the $\Upsilon$ and $\Upsilon^{\prime} A$ dependence within their uncertainties [48]. Additionally the $\psi^{\prime} / \psi$ ratio appears to be independent of $A$ [27] but the data is such that a size-dependence of the absorption cross section cannot be ruled out. At the E772 energy, $\sqrt{s}=38.8$ $\mathrm{GeV}$, the target momentum fractions probed are $x \leq 0.08$ for the charmonium states, just in the shadowing region, while for the $\Upsilon$ states, $x \leq 0.25$, in the EMC or antishadowing regions. At the lower energies of other $J / \psi$ measurements, shadowing should be less effective than absorption [55]. The mass scale in the PDF is also important if the shadowing is $Q^{2}$ dependent [56]. In $Q^{2}$-dependent shadowing models, the gluon distribution evolves fastest with $Q^{2}$; the valence and sea quark evolution is rather weak for $2<Q^{2}<10$ $\mathrm{GeV}^{2}$ [49, 56. We note that nuclear deep-inelastic scattering data do not exhibit strong $Q^{2}$ dependence for $Q^{2} \leq 100 \mathrm{GeV}^{2}$ [57], in agreement with these models, but it is more difficult to probe the $Q^{2}$ dependence of gluon shadowing. Precision $p A$ data is needed to sort out these effects, particularly at higher than current energies since at RHIC and LHC we will be in a new regime where $x$ is small and $Q^{2}$ is relatively large. Measurements of dilepton production will prove helpful, as we discuss later, since the dilepton continuum could be influenced by shadowing but not by absorption.

The $p_{T}$-dependence of the suppression from shadowing/absorption is especially crucial for out later considerations. Since $p A$ studies are not possible at the LHC, the exact rapidity and $p_{T}$-dependence of shadowing/absorption suppression at the low $x$ values probed at $5.5 \mathrm{TeV}$ will be imprecisely known. Thus, it is fortunate that the $p_{T}$-dependence of such suppression should be much weaker than that from QGP suppression (as we shall discuss below). As noted above, if shadowing is dominant, then suppression from this purely nuclear source should also be essentially independent of the Upsilon state. As our ensuing discussion will show, this would be a very important advantage. In contrast, if absorption is dominant the magnitude of the suppression could depend on the Upsilon state. Finally, we note that any broadening of the $p_{T}$-dependence due to initial-state interactions in the nuclear target [58] may be expected to be negligible. Thus, we believe that any alteration of the 


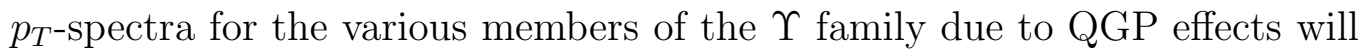
stand out clearly. Even more crucially, in the absence of QGP suppression the ratios of rates for different $\Upsilon$ family members should exhibit $p_{T}$ dependence that is precisely that predicted without including shadowing or absorption. The $p_{T}$-dependence for such ratios is then a direct probe of the QGP physics.

\section{Sensitivity of quarkonium suppression to the QGP model}

As we have just shown, the $\Upsilon$ production rate is high enough at the LHC for any QGP effects on the $\Upsilon$ family to be measurable. Our discussion in Sec. 2 suggested that at the high temperatures predicted for the minijet plasma, $\Upsilon$ production may be suppressed at the LHC. We would like to use this suppression to determine the initial conditions of the QGP, including $T_{0}, t_{0}$, and the temperature dependence of the QGP screening mass $\mu(T)$. A simple measurement of the overall suppression of the production rates for the Upsilon family members will not be adequate. We must consider additional observables that could be affected by the presence of the QGP. In this section, we will show that the $p_{T}$-dependence of the suppression provides a remarkable amount of information.

If the QGP were of infinite temporal and spatial extent, resonance production would be suppressed as long as $T>T_{D}$. But, there would be no significant $p_{T}$-dependence. In fact, however, both the lifetime and the spatial extent of the QGP are finite. Since a color singlet $Q \bar{Q}$ pair takes some time to form a bound state, the competition between the formation time, the time needed for the $Q \bar{Q}$ to separate to the bound state radiusף, and the characteristics of the plasma all affect the $p_{T}$-dependence of the $\Upsilon$ states. We will now discuss the sensitivity of the onium suppression patterns as a function of $p_{T}$ to these ingredients.

The finite lifetime of the plasma sets an upper limit on the $p_{T}$ at which the onium states can be suppressed. In the plasma rest frame, the $b \bar{b}$ forms a bound state in the time $t_{F}=\gamma \tau_{F}$ where $\gamma=\sqrt{1+\left(p_{T} / M\right)^{2}}$ ( $M$ is the onium mass) and the formation times, $\tau_{F}$, for the bottonium and charmonium states were given in Table 2. When the plasma has cooled below $T_{D}$, the production of the bound state is no longer suppressed. The time at which this occurs can be determined from the Bjorken model [59] which assumes that the evolution of the system is described by ideal hydrodynamics, cooling by an isentropic

\footnotetext{
${ }^{6}$ If absorption is significant and $\sigma_{\text {abs }}$ depends upon the Upsilon state, then the ratio of $p_{T}$-integrated cross sections would be affected but not the $p_{T}$ variation.

${ }^{7}$ If the $Q \bar{Q}$ is produced as a color octet, it is less likely to produce quarkonium than heavy-flavored hadrons.
} 
expansion so that

$$
s_{D} t_{D}=s_{0} t_{0}
$$

Since the entropy density, $s$, is proportional to $T^{3}$, the time at which the temperature drops below $T_{D}$ is

$$
t_{D}=t_{0}\left(\frac{T_{0}}{T_{D}}\right)^{3} .
$$

As long as $t_{D} / t_{F}>1$, quarkonium formation will be suppressed. Thus the maximum $p_{T}$ at which suppression occurs is

$$
p_{T, \max }=M \sqrt{\left(t_{D} / \tau_{F}\right)^{2}-1} .
$$

The values for $t_{D}$ and $p_{T, \text { max }}$ for all the onium states are given in Tables 5 and 6 for both forms of $\mu(T)$, eqs. (5) and (6), and three different sets of initial conditions for LHC and RHIC energies.

It is easy to check whether or not the bottonium states are suppressed given the formation time and $T_{0}$ from the minijet initial conditions. The $\mathrm{SU}(N)$ parameterization of eq. (5), with $T_{c}=260 \mathrm{MeV}$, results in $t_{D} / \tau_{F}>$ 1 for all the members of the Upsilon family. Thus, all the states will be suppressed up to rather large values of $p_{T}$, suggesting that any suppression should be easily observable within the $p_{T}$ acceptance of CMS. However, $T_{0}$ is reduced to $900 \mathrm{MeV}$ when quark degrees of freedom are added as in the 3Flavor scenario of eq. (6); the result is that $t_{D} / \tau_{F}<1$ for all the bottonium states and no suppression occurs. In other words, if the 3-Flavor quarkgluon plasma is finite and quickly equilibrated, none of the $\Upsilon$ states will be suppressed, and the $p_{T}$-dependence should be unchanged from $p p$ production modulo any nuclear effects on $\Upsilon$ production.

In contrast, the charmonium states are suppressed in both the $\mathrm{SU}(N)$ and the 3-Flavor $\mu(T)$ scenarios. The $\mathrm{SU}(N)$ case gives values of $p_{T, \max }$ similar to or smaller than those of the bottonium family because of the smaller charmonium masses. This should be contrasted with the 3-Flavor QGP for which the $J / \psi$ would be suppressed over a very narrow range of $p_{T}$ relative to the $\chi_{c}$ and $\psi^{\prime}$ since the value of $T_{D}$ for the $J / \psi$ is nearly twice that of the other charmonium states (see Table 3 for $T_{D}$ ). If the $p_{T}$-dependence of the nuclear effects on the charmonium states is weak, then the ratio of higher charmonium states to the $J / \psi$ as a function of $p_{T}$ would still be useful.

We discuss the other initial conditions shown in Tables 5 and 6 and their consequences later.

In a finite system, such as those produced in heavy-ion collisions, the suppression also depends on the size of the system. We present a schematic 


\begin{tabular}{|c|c|c|c|c|}
\hline \multicolumn{5}{|c|}{$\overline{\mathrm{LHC}}$} \\
\hline & \multicolumn{2}{|c|}{ 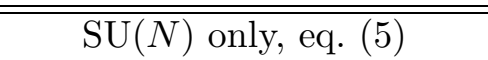 } & \multicolumn{2}{|c|}{ "gluons and quarks, eq. (6) } \\
\hline \multicolumn{5}{|c|}{ minijet initial conditions } \\
\hline & \multicolumn{2}{|c|}{$T_{0}=1.14 \mathrm{GeV}, t_{0}=0.1 \mathrm{fm}$} & \multicolumn{2}{|c|}{$T_{0}=900 \mathrm{MeV}, t_{0}=0.1 \mathrm{fm}$} \\
\hline & $t_{D}(\mathrm{fm})$ & $p_{T, \max }(\mathrm{GeV})$ & $t_{D}(\mathrm{fm})$ & $p_{T, \max }(\mathrm{GeV})$ \\
\hline$\Upsilon$ & 2.49 & 29.5 & 0.105 & 0 \\
\hline$\overline{\Upsilon^{\prime}}$ & 8.474 & 43.5 & 1.67 & 0 \\
\hline$\chi_{b}$ & 8.474 & 30.7 & 1.46 & 0 \\
\hline$\psi$ & 8.474 & 29.0 & 1.54 & 4.34 \\
\hline$\psi^{\prime}$ & 8.474 & 20.6 & 15.7 & 38.6 \\
\hline$\chi_{c}$ & 8.474 & 14.4 & 17.5 & 30.5 \\
\hline \multicolumn{5}{|c|}{ Uncertainty relation initial conditions } \\
\hline \multicolumn{5}{|c|}{$C=1$} \\
\hline & \multicolumn{2}{|c|}{$T_{0}=600 \mathrm{MeV}, t_{0}=0.11 \mathrm{fm}$} & \multicolumn{2}{|c|}{$T_{0}=785 \mathrm{MeV}, t_{0}=0.084 \mathrm{fm}$} \\
\hline \multicolumn{5}{|c|}{$C=3$} \\
\hline & \multicolumn{2}{|c|}{$T_{0}=346 \mathrm{MeV}, t_{0}=0.57 \mathrm{fm}$} & \multicolumn{2}{|c|}{$T_{0}=453 \mathrm{MeV}, t_{0}=0.436 \mathrm{fm}$} \\
\hline & $t_{D}(\mathrm{fm})$ & $p_{T, \max }(\mathrm{GeV})$ & $t_{D}(\mathrm{fm})$ & $p_{T, \max }(\mathrm{GeV})$ \\
\hline$\Upsilon$ & 0.39 & 0 & 0.03 & 0 \\
\hline$\Upsilon^{\prime}$ & 1.3 & 0 & 0.455 & 0 \\
\hline$\chi_{b}$ & 1.3 & 0 & 0.82 & 0 \\
\hline$\psi$ & 1.3 & 3.27 & 0.42 & 0 \\
\hline$\psi^{\prime}$ & 1.3 & 0 & 4.28 & 9.9 \\
\hline$\chi_{c}$ & 1.3 & 0 & 4.78 & 7.6 \\
\hline \multicolumn{5}{|c|}{ parton gas initial conditions } \\
\hline & \multicolumn{2}{|c|}{$T_{0}=820 \mathrm{MeV}, t_{0}=0.5 \mathrm{fm}$} & \multicolumn{2}{|c|}{$T_{0}=820 \mathrm{MeV}, t_{0}=0.5 \mathrm{fm}$} \\
\hline & $t_{D}(\mathrm{fm})$ & $p_{T, \max }(\mathrm{GeV})$ & $t_{D}(\mathrm{fm})$ & $p_{T, \max }(\mathrm{GeV})$ \\
\hline$\Upsilon$ & 4.6 & 56.53 & 0.19 & 0 \\
\hline$\overline{\Upsilon^{\prime}}$ & 15.69 & 81.98 & 3.09 & 12.83 \\
\hline$\chi_{b}$ & 15.69 & 58.9 & 5.53 & 18.58 \\
\hline$\psi$ & 15.69 & 54.0 & 2.85 & 9.34 \\
\hline$\psi^{\prime}$ & 15.69 & 38.5 & 29.1 & 71.6 \\
\hline$\chi_{c}$ & 15.69 & 27.2 & 32.5 & 56.76 \\
\hline
\end{tabular}

Table 5: LHC values of $t_{D}$, and $p_{T, \max }$ for the two choices, eq. (5) and eq. (6), of $\mu(T)$, and for three sets of initial conditions. 


\begin{tabular}{|c|c|c|c|c|}
\hline \multicolumn{5}{|c|}{$\mathrm{RHIC}$} \\
\hline & \multicolumn{2}{|c|}{$\mathrm{SU}(N)$ only, eq. (5) } & \multicolumn{2}{|c|}{ gluons and quarks, eq. (6) } \\
\hline \multicolumn{5}{|c|}{ minijet initial conditions } \\
\hline & \multicolumn{2}{|c|}{$T_{0}=445 \mathrm{MeV}, t_{0}=0.1 \mathrm{fm}$} & \multicolumn{2}{|c|}{$T_{0}=360 \mathrm{MeV}, t_{0}=0.1 \mathrm{fm}$} \\
\hline & $t_{D}(\mathrm{fm})$ & $p_{T, \max }(\mathrm{GeV})$ & $t_{D}(\mathrm{fm})$ & $p_{T, \max }(\mathrm{GeV})$ \\
\hline$\Upsilon$ & 0.15 & 0 & 0.003 & 0 \\
\hline$\Upsilon^{\prime}$ & 0.5 & 0 & 0.05 & 0 \\
\hline$\chi_{b}$ & 0.5 & 0 & 0.094 & 0 \\
\hline$\psi$ & 0.5 & 0 & 0.048 & 0 \\
\hline$\psi^{\prime}$ & 0.5 & 0 & 0.493 & 0 \\
\hline$\chi_{c}$ & 0.5 & 0 & 0.55 & 0 \\
\hline \multicolumn{5}{|c|}{ Uncertainty relation initial conditions } \\
\hline \multicolumn{5}{|c|}{$C=1$} \\
\hline & \multicolumn{2}{|c|}{$T_{0}=400 \mathrm{MeV}, t_{0}=0.164 \mathrm{fm}$} & \multicolumn{2}{|c|}{$T_{0}=524 \mathrm{MeV}, t_{0}=0.126 \mathrm{fm}$} \\
\hline \multicolumn{5}{|c|}{$C=3$} \\
\hline & \multicolumn{2}{|c|}{$T_{0}=231 \mathrm{MeV}, t_{0}=0.85 \mathrm{fm}$} & \multicolumn{2}{|c|}{$T_{0}=303 \mathrm{MeV}, t_{0}=0.65 \mathrm{fm}$} \\
\hline & $t_{D}(\mathrm{fm})$ & $p_{T, \max }(\mathrm{GeV})$ & $t_{D}(\mathrm{fm})$ & $p_{T, \max }(\mathrm{GeV})$ \\
\hline$\Upsilon$ & 0.17 & 0 & 0.013 & 0 \\
\hline$\Upsilon^{\prime}$ & 0.59 & 0 & 0.2 & 0 \\
\hline$\chi_{b}$ & 0.59 & 0 & 0.364 & 0 \\
\hline$\psi$ & 0.59 & 0 & 0.188 & 0 \\
\hline$\psi^{\prime}$ & 0.59 & 0 & 1.92 & 2.94 \\
\hline$\chi_{c}$ & 0.59 & 0 & 2.14 & 1.31 \\
\hline \multicolumn{5}{|c|}{ parton gas initial conditions } \\
\hline & \multicolumn{2}{|c|}{$T_{0}=550 \mathrm{MeV}, t_{0}=0.7 \mathrm{fm}$} & \multicolumn{2}{|c|}{$T_{0}=550 \mathrm{MeV}, t_{0}=0.7 \mathrm{fm}$} \\
\hline & $t_{D}(\mathrm{fm})$ & $p_{T, \max }(\mathrm{GeV})$ & $t_{D}(\mathrm{fm})$ & $p_{T, \max }(\mathrm{GeV})$ \\
\hline$\Upsilon$ & 1.95 & 22.3 & 0.082 & 0 \\
\hline$\Upsilon^{\prime}$ & 6.63 & 33.4 & 1.3 & 0 \\
\hline$\chi_{b}$ & 6.63 & 23.2 & 2.33 & 0 \\
\hline$\psi$ & 6.63 & 22.6 & 1.2 & 2.8 \\
\hline$\psi^{\prime}$ & 6.63 & 15.9 & 12.3 & 30.12 \\
\hline$\chi_{c}$ & 6.63 & 11.1 & 13.7 & 23.75 \\
\hline
\end{tabular}

Table 6: RHIC values of $t_{D}$, and $p_{T, \max }$ for the two choices, eq. (5) and eq. (6), of $\mu(T)$, and for three sets of initial conditions. 
calculation of the $p_{T}$-dependence of the suppression following Ref. [60]. Similar analyses of QGP production have also been done 22, 61, 62]. We can assume that the initial entropy profile is dependent on the radius of the QGP,

$$
s_{0}(r)=s_{0}\left(1-\left(\frac{r}{R}\right)^{2}\right)^{\beta},
$$

so that

$$
t_{D}(r)=t_{D}(0)\left(1-\left(\frac{r}{R}\right)^{2}\right)^{\beta}
$$

where $t_{D}(0)$ is the value of $t_{D}$ calculated in eq. (16) for resonances produced in the center of the system and $R$ is the transverse radius of the plasma, assumed to be no larger than the radius of the nucleus, $R \simeq 1.2 A^{1 / 3}$. At any time $t$ during the evolution of the QGP, the spatial boundary of the screening region is located at the transverse radius $r_{S}$ given by $t_{D}\left(r_{S}\right)=t$,

$$
r_{S}=R\left(1-\left(\frac{t}{t_{D}(0)}\right)^{1 / \beta}\right)^{1 / 2}
$$

In general, if the $Q \bar{Q}$ pair is created with transverse position $x^{\mu}=(0, \mathbf{r}, 0)$ and momentum $p^{\mu}=\left(\sqrt{M^{2}+p_{T}^{2}}, \mathbf{p}_{\mathbf{T}}, 0\right)$, at the formation time $\tau_{F}$ the pair will form a bound state at $x^{\mu}=\left(\tau_{F} \sqrt{1+\left(p_{T} / M\right)^{2}}, \mathbf{r}+\tau_{F} \mathbf{p}_{\mathbf{T}} / M, 0\right)$. If $\mid \mathbf{r}+$ $\tau_{F} \mathbf{p}_{\mathbf{T}} / M \mid \geq r_{S}$, the $Q \bar{Q}$ pair escapes the QGP before $\tau_{F}$ and will successfully form bound states. This inequality will be satisfied (and the pairs will escape) for a range of angles between $\mathbf{r}$ and $\mathbf{p}_{\mathbf{T}}-0 \leq \theta \leq \theta_{\max }\left(r, p_{T}\right)-$ provided $p_{T}$ is such that $\left(r+\tau_{F} p_{T} / M\right)>r_{S}$. Here, $\theta_{\max }$ also depends on $\tau_{F}, M$, and $r_{S}$ as detailed below. The $p_{T}$-dependent probability that the $Q \bar{Q}$ pair survives and is able to form an onium state, $S\left(p_{T}\right)$, is the ratio of the number of bound states produced by those pairs that escape the plasma to the maximum possible number of onium bound states that would be formed at the given $p_{T}$ in the absence of the QGP:

$$
S\left(p_{T}\right)=\frac{\left.\int_{0}^{R} d r r \rho(r) \theta_{(} r, p_{T}\right)}{\pi \int_{0}^{R} d r r \rho(r)},
$$

where we parameterize $\rho(r)=\left(1-(r / R)^{2}\right)^{\alpha}$ and

$$
\theta\left(r, p_{T}\right)= \begin{cases}\pi & z \leq-1 \\ \cos ^{-1} z & |z|<1 \\ 0 & z \geq 1\end{cases}
$$


where

$$
z=\frac{r_{S}^{2}-r^{2}-\left(\frac{\tau_{F} p_{T}}{M}\right)^{2}}{2 \tau_{F} r \frac{p_{T}}{M}} .
$$

When $z \geq 1$ the pair cannot escape, whereas when $z \leq-1$ the pair always escapes.

The results for the survival probability $S\left(p_{T}\right)$, with $\alpha=1 / 2$ and $\beta=1 / 4$, are shown in Figs. $\beta$ (a), 负(b), and 3 (c) for the $T_{D}$ values implied by eq. (5) (see Table 3) and for a decreasing sequence of radii: (a) $R=R_{\mathrm{Pb}}=7.1 \mathrm{fm}$, (b) $R=R_{\mathrm{S}}=3.8 \mathrm{fm}$ and (c) $R=1 \mathrm{fm}$, respectively. In all three cases, we observe that $S\left(p_{T}\right) \sim 0$, corresponding to essentially total suppression, at small $p_{T}$ with our assumption of an equilibrated system. For $R=R_{\mathrm{Pb}}$ the system is large enough for the finite lifetime to be more important than its finite size. Therefore $S\left(p_{T}\right)=1$ for $p_{T} \geq p_{T, \max }$ while states with $p_{T}<p_{T, \text { max }}$ are suppressed. If the QGP is of smaller spatial extent, the survival probability can become unity for $p_{T}<p_{T, \text { max }}$ since a smaller $p_{T}$ is sufficient for the pair to escape the system. This is illustrated by the results for $R=R_{\mathrm{S}}$ and $R=1$ fm displayed in (b) and (c) of Fig. 3. As $R$ decreases, the largest $p_{T}$ value for which suppression occurs decreases more rapidly for the $\Upsilon^{\prime}$ and $\chi_{b}$ states than for the $\Upsilon$ because of their larger breakup time, $t_{D}$; see Table 5 . In fact, when $R=1 \mathrm{fm}, S\left(p_{T}\right)=1$ for the $\Upsilon^{\prime}$ at $p_{T} \approx 10 \mathrm{GeV}$ while the $\Upsilon$ remains suppressed up to $p_{T} \approx 20 \mathrm{GeV}$, as shown in Fig. 3(c).

We should compare these results to other possible QGP scenarios.

- First, there is the possibility that we have overestimated the amount of screening that occurs for a given level of minijet production. As we have noted, see Table 5, the screening times, $t_{D}$, are generally shorter for the perturbative estimate of eq. (6) [even if quarks are not included by setting $n_{f}=0$ ] than for the $\mathrm{SU}(N)$ gauge parameterization given in eq. (5) 8 . The result is that there is no suppression of the $\Upsilon$ states for the 3-Flavor QGP in equilibrium if minijet initial conditions apply.

- It is also possible that the minijet density is different than we assumed in our calculations. For example, if the appropriate minijet momentum scale, $p_{0}$, were larger (smaller) than the value of $p_{0}=2 \mathrm{GeV}$ assumed above, $t_{0}$ and, consequently, the minijet density, $T_{0}$ and, from eq. (16), $t_{D}$ would all be smaller (larger). A lower minijet density could produce a different suppression pattern for the $\Upsilon$ family; e.g. a choice of $p_{0}$ is possible such that $\Upsilon$ production is not suppressed, while $\Upsilon^{\prime}$ and $\chi_{b}$ production are.

\footnotetext{
${ }^{8}$ The $\psi^{\prime}$ and $\chi_{c}$ are an exception because the lower value of $T_{c}$ found when quarks are included reduced the breakup temperature, resulting in a longer screening time.
} 

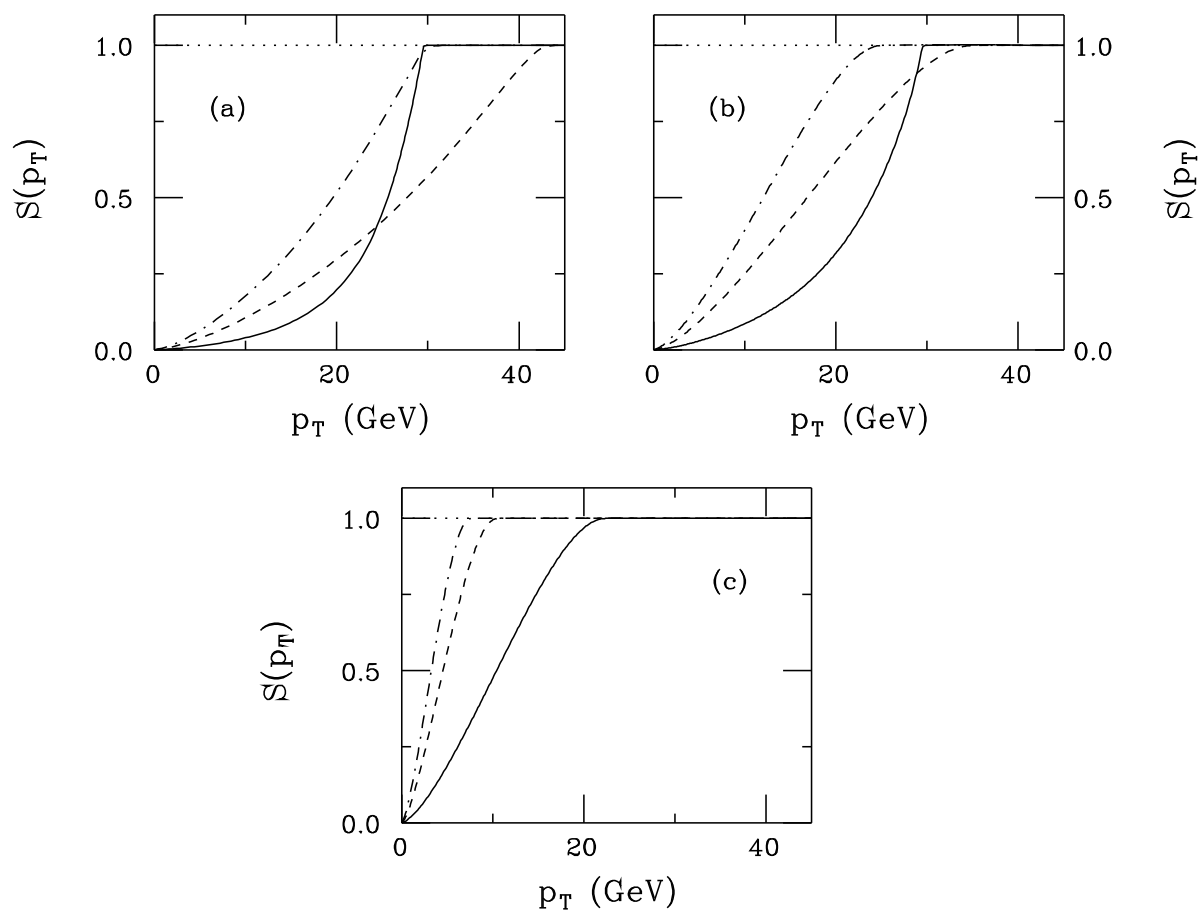

Figure 3: For the $\mathrm{SU}(N) \mu(T)$ of eq. (5) and the associated minijet initial conditions of $T_{0}=1.14 \mathrm{GeV}, t_{0}=0.1 \mathrm{fm}$, the suppression factor $S\left(p_{T}\right)$, calculated according to eq. (21), is shown for $\Upsilon$ (solid), $\Upsilon^{\prime}$ (dashed), and $\chi_{b}$ (dot-dashed) production in three cases: (a) $R=R_{\mathrm{Pb}}$; (b) $R=R_{\mathrm{S}}$; and (c) $R=1 \mathrm{fm}$.

- We note that typical early estimates of the initial conditions without minijet production, $t_{0}=1 \mathrm{fm}$ and $T_{0}=250-300 \mathrm{MeV}$ at the LHC (see e.g. [12]), produce no bottonium suppression regardless of the form of $\mu(T)$.

- An alternative set of initial conditions is obtained using the uncertainty principle [63, 64]. In this case, $t_{0}\langle E\rangle \sim C \hbar c$, with $\langle E\rangle \sim 3 T_{0}$ for a thermal average. In previous calculations, $C=1$ and 3 were chosen. Those studies, using $T_{c}=200 \mathrm{MeV}$ and assuming a 3-Flavor QGP, obtained $T_{0}=0.46 \mathrm{GeV}$ and $t_{0}=0.42 \mathrm{fm}$ for $C=3$ and $T_{0}=0.81$ $\mathrm{GeV}$ and $t_{0}=0.08 \mathrm{fm}$ for $C=1$ [64]. In Tables 5 and 6 we update these results for the pure gluon $\mathrm{SU}(N)$ and 3-Flavor QGP cases, using the appropriate $T_{c}$ for each. As can be seen from the formulae of Ref. [64], the 3-Flavor scenario has a higher $T_{0}$ than the $\mathrm{SU}(N)$ case given our assumption that the final multiplicity is the same regardless of the initial conditions. We note that either choice of $C$ produces 
the same results because $T_{0} \propto \sqrt{3 / C}$ (as shown in 64) and $t_{0} T_{0} \propto$ $C / 3$, implying that the $C$ dependence cancels in the expression for $t_{D}$, eq. (16). Neither scenario leads to any bottonium suppression, and suppression of the charmonium states is only possible for rather low $p_{T}$ values.

- The final initial condition scenario that we discuss is based on a parton gas model as adopted for $J / \psi$ suppression at RHIC and LHC energies in Ref. [65]. In this model one finds that the kinetic equilibration time is longer than for any of the cases discussed so far, $t_{0} \sim 0.5-$ $0.7 \mathrm{fm}$. This time is reached when the momentum distributions are locally isotropic due to elastic scatterings and the expansion of the system. Chemical equilibrium is generally not assumed but the system moves toward equilibrium as a function of time. In this picture, the cooling of the plasma is more rapid than the simple Bjorken scaling picture [59] we have adopted, producing incomplete suppression at low $p_{T}$. To make a schematic comparison with our assumption of complete equilibrium, we give in Tables 5 and 6 the values of $t_{D}$ and $p_{T, \text { max }}$ for the onium states computed using the same $T_{0}$ and $t_{0}$ as employed in their calculation, derived from the HIJING Monte Carlo code 66. (Note that we use the same initial values independent of the form assumed for $\mu(T)$.) Because the equilibration time of the parton gas is longer than that obtained from the minijet initial conditions, the time the system spends above the breakup temperature is also longer, leading to suppression for both the $\mathrm{SU}(N)$ and the 3-Flavor forms of $\mu(T)$, even though $T_{0}$ is lower. These results are illustrated in Fig. 6 for the two $\mu(T)$ parameterizations. Figs. 4 (a) and 4 (b) illustrate the survival probability $S\left(p_{T}\right)$ for the $\mathrm{SU}(N)$ plasma assuming $R=R_{\mathrm{Pb}}$ and $1 \mathrm{fm}$, respectively. In the $\mathrm{SU}(N)$ case, $p_{T, \max }$ is sufficiently large that the lead nucleus is already small enough for the $p_{T}$ range of the suppression to be less than the calculated $p_{T, \max }$. The 3-Flavor QGP results are shown in Figs. 1 (c) and $4(\mathrm{~d})$ for $R=R_{\mathrm{Pb}}$ and $1 \mathrm{fm}$, respectively. Here the temperature is not high enough for $\Upsilon$ suppression but the $\Upsilon^{\prime}$ and $\chi_{b}$ are suppressed, albeit over a much smaller $p_{T}$ range than in the $\mathrm{SU}(N)$ case.

A given prediction for the survival probability $S\left(p_{T}\right)$ can be used to calculate the $p_{T}$ distributions of the $\Upsilon$ states. Since $S\left(p_{T}\right)$ depends upon the $T_{D}$ and $\tau_{F}$ of each Upsilon resonance, each state will have a unique $p_{T}$ dependence, despite the fact that the $p_{T}$ distributions determined from the color evaporation model are the same for directly produced $\Upsilon, \Upsilon^{\prime}$ and $\chi_{b}$ before inclusion of QGP effects. As we have noted, the precise $S\left(p_{T}\right)$ factors for the different $\Upsilon$ resonances also depend on the choice of $\mu(T), t_{0}, T_{0}$, and $R$. 

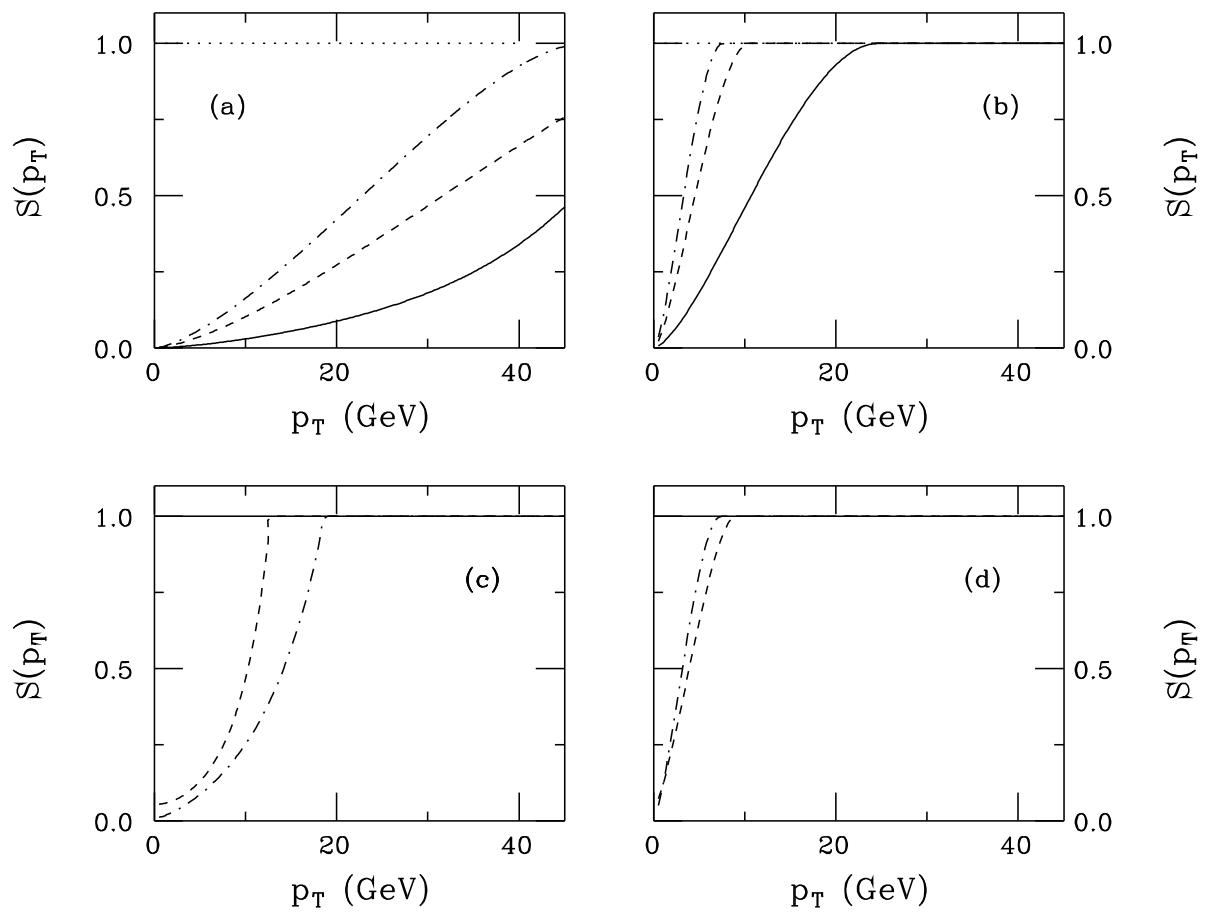

Figure 4: For parton gas initial conditions of $T_{0}=820 \mathrm{MeV}, t_{0}=0.5 \mathrm{fm}$, the suppression factor $S\left(p_{T}\right)$, calculated according to eq. (21), is shown for $\Upsilon$ (solid), $\Upsilon^{\prime}$ (dashed), and $\chi_{b}$ (dot-dashed) production in four cases: (a) $R=R_{\mathrm{Pb}}$ and $\mu(T)$ of eq. (5); (b) $R=1 \mathrm{fm}$ and $\mu(T)$ of eq. (5); (c) $R=R_{\mathrm{Pb}}$ and $\mu(T)$ of eq. (可); (d) $R=1 \mathrm{fm}$ and $\mu(T)$ of eq. (6).

The resulting $p_{T}$ spectra are shown in Fig. 5 in the case of $S\left(p_{T}\right)$ taken from Fig. 3(a), assuming the $\mathrm{SU}(N)$ form of $\mu(T)$, with $R=R_{\mathrm{Pb}}, t_{0}=0.1 \mathrm{fm}$ and $T_{0}=1.14 \mathrm{GeV}$. The upper curves are the $p_{T}$ distributions in $p p$ collisions at $\sqrt{s}=5.5 \mathrm{TeV}$ while the lower ones are the $p p$ curves multiplied by $S\left(p_{T}\right)$ to indicate the $p_{T}$ distribution expected in the presence of the quarkgluon plasma. Note that plasma production decreases the slope of the $p_{T}$ distribution.

The raw $p_{T}$ distributions before including the survival probabilities were obtained using the color evaporation model predictions calculated with the next-to-leading order $Q \bar{Q}$ code of Ref. [26]. It is well-known that at low $p_{T}$ it is necessary to resum the next-to-leading $\ln \left(M^{2} / p_{T}^{2}\right)$ powers for each power of $\alpha_{s}$ to get an exponential factor. This has been extensively studied for $W$ and $Z$ production [67, 68, 69, 70, 71, 72]. The procedure involves computing a form factor in impact parameter $(b)$ space, and then Fourier transforming back to $p_{T}$ space. For $\Lambda_{\mathrm{QCD}} \ll 1 / b<M$, the form factor can be computed perturbatively; for $b \gtrsim 1 / \Lambda_{\mathrm{QCD}}$ non-perturbative 

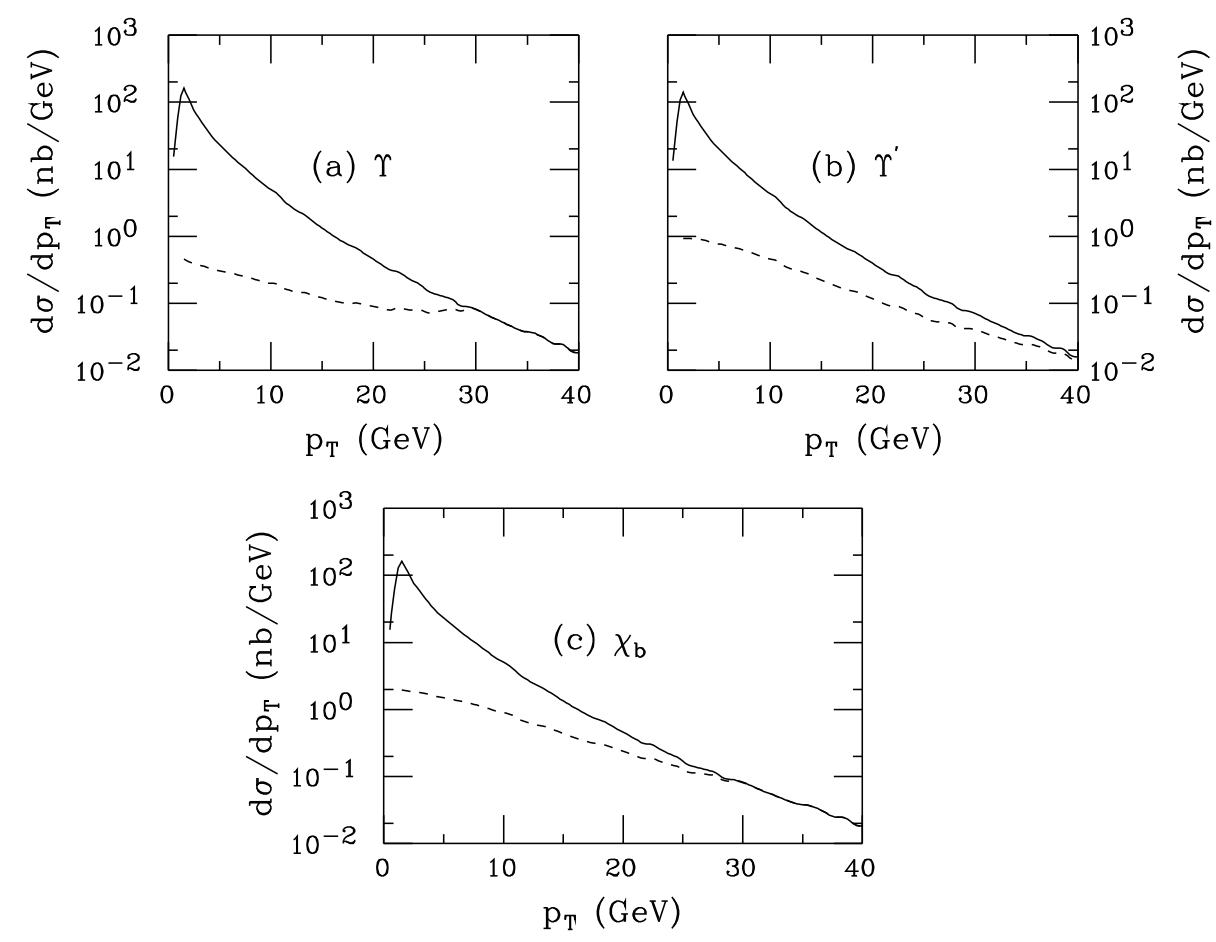

Figure 5: The $p_{T}$ distributions for (a) $\Upsilon$, (b) $\Upsilon^{\prime}$, and (c) $\chi_{b}$ production are given for $p p$ interactions (solid) and plasma production (dashed). The plasma results are the $p p$ distributions multiplied by $S\left(p_{T}\right)$ as computed for $R=R_{\mathrm{Pb}}, t_{0}=0.1 \mathrm{fm}$ and $T_{0}=1.14 \mathrm{GeV}$ and assuming the $\mathrm{SU}(N)$ form of $\mu(T)$ as given in eq. (5); $S\left(p_{T}\right)$ for this case was plotted in Fig. 3(a). We have taken $\sqrt{s}=5.5 \mathrm{TeV}$. The differential cross sections (before including $S\left(p_{T}\right)$ ) are normalized to the integrated values tabulated in Table 4 .

modeling is necessary. In the latter range, the conventional procedure is to introduce a cutoff in $b$ and a non-perturbative factor in the $b$-space form factor. It is found $[72]$ that the non-perturbative component dominates the $p_{T}$-dependence modifications for $p_{T} \lesssim 6 \mathrm{GeV}$, implying substantial uncertainties for $p_{T}$-dependence in this domain. However, Ref. 72 also demonstrates that the ratio $\left[d \sigma(W) / d p_{T}\right] /\left[d \sigma(Z) / d p_{T}\right]$ is very insensitive to the non-perturbative uncertainties due to the fact that $m_{W}$ is not very different from $m_{Z}$. Further, the ratio deviates only slightly from the perturbatively computed value. Applied to the closely-spaced resonances of the Upsilon family, the formalism of Ref. [72] implies that the color evaporation model predictions for the ratios of the production rates for different $\Upsilon$ states will also not be significantly modified by the non-perturbative physics. The ability to reliably compute a ratio such as $\Upsilon^{\prime} / \Upsilon$ as a function of $p_{T}$ down to relatively 
low $p_{T}$ values is critical to the program described in the next section.

\section{A program for determining the nature of the QGP}

We have seen that the uncertainties in the initial conditions as reflected in $t_{0}$ and $T_{0}$, the uncertainty in the QGP radius $R$, and the ambiguity in the temperature dependence of the screening mass, $\mu(T)$, all imply that there are many possible results for the relative suppression of the $\Upsilon$ states. The challenge will be to extract the correct plasma picture from the data. We describe below the inputs, procedures and detector features required in order that the production and suppression of the $\Upsilon$ states can be used as a tool to determine the true state of the plasma and the proper initial conditions. Data from the LHC and RHIC will prove complementary; it is likely that Upsilon family production rates must be measured at both machines in order to reach reliable conclusions. Further, measurements of the charmonium states at both LHC and RHIC will provide valuable information. We organize the following discussion into a series of (overlapping) tasks and requirements necessary to extract information about the QGP.

Checking the color evaporation model and establishing a baseline.

As a first step, the production rates and $p_{T}$ spectra for the $\Upsilon$ states must be measured in $p p$ collisions at the standard LHC nucleon-nucleon collision energy of $5.5 \mathrm{TeV}$ and, by lowering the energy of the LHC still further, at 1.8 TeV.9 This will allow us to check the predictionst of the color evaporation model. In particular, we must confirm that the the $\Upsilon^{\prime} / \Upsilon$ and $\Upsilon^{\prime \prime} / \Upsilon$ ratios are independent of energy and $p_{T}$ and that the (common) $p_{T}$-dependence of the individual cross sections agrees with the color evaporation prediction. These measurements will allow us to confirm our perturbative modeling and understanding of $\Upsilon$ production in the clean $p p$ environment, thus establishing a baseline for comparison with results obtained for nuclear collisions.

\section{Determining the effects of shadowing and nucleon absorption using RHIC data.}

Next, we must determine if the $\Upsilon^{\prime} / \Upsilon$ ratio is independent of purely nuclear matter effects (e.g. shadowing and absorption) by measuring the ratio

\footnotetext{
${ }^{9}$ The lower energy measurement should agree with that obtained at the Tevatron 36] to the extent that valence quark distribution function contributions do not contribute significantly in $p \bar{p}$ collisions, but a direct $p p$ measurement will remove the necessity of making this assumption in order to carry out the following program.

${ }^{10}$ Assuming that gluon fusion is dominant at both LHC and Tevatron energies.
} 
in $p A$ collisions. Since these studies are not possible at the LHC, this must be established at RHIC. The heaviest nuclear beam at RHIC will be gold, but the shadowing should be quite similar in gold and lead. Since RHIC is a dedicated heavy-ion facility, the PHENIX experiment at RHIC is expected to obtain several thousand $\Upsilon$ events per year along with a similar number of $\Upsilon^{\prime}$ 's 73 . This should be sufficient to determine: a) the $p_{T}$ dependence of the $\Upsilon^{\prime} / \Upsilon$ ratio in $p A$ interactions where nuclear effects are present but no QGP is expected; and b) the magnitude of the $\Upsilon^{\prime} / \Upsilon$ ratio. If the ratio is the same (smaller) than in $p p$ collisions, then shadowing (absorption) is probably the dominant purely nuclear effect. The dependence of the onium ratios on shadowing/absorption may also be established by studying $\psi^{\prime} / \psi$ in $p A$ collisions: lower $x$ partons are probed and the rates are higher.

If shadowing is dominant over absorption, then measurements of the actual magnitude of the gluon shadowing will be important if we are to use the absolute $p_{T}$-dependence of the onium cross sections instead of their ratios to analyze the QGP. It has been suggested that $p A$ measurements of the dilepton continuum $(M \geq 1 \mathrm{GeV})$ at RHIC will provide a measure of gluon shadowing since dileptons from charm production and decay (mainly from gluon fusion) will be the largest contribution in the mass region below the $J / \psi$ 74. Such charm studies are particularly useful because they probe gluon shadowing at the low $x$ values that will be important for Upsilon production at the LHC. Measurements at RHIC for dilepton masses near the $\Upsilon$ mass probe parton $x$ values of $x \sim 0.1$ (at $y \sim 0$ ), i.e. substantially above those appropriate at the LHC and also in a range where little shadowing is expected [75]. In order to probe small $x$ values for dilepton masses near $M_{\Upsilon}$, it would be necessary to accumulate good statistics for the $\Upsilon$ 's at high rapidity, which can be observed in the muon arms of the detector. An $\Upsilon$ with $y \sim+2$ or -2 will have $x_{2} \sim 0.35$, i.e. in the EMC region, or $x_{2} \sim 0.006$, in the shadowing region, respectively. The latter would probe nuclear effects on $\Upsilon$ production for gluon $x$ values that are dominant for $\Upsilon$ production at the LHC around $y \sim 0$.

Perhaps the best way to determine the importance of nucleon absorption for the onium states is to measure the relative rapidities of the onium states and the initial nucleons since the baryon number in the central region of nuclear collisions at RHIC and LHC is expected to be small. If the nucleons and quarkonium states are far apart in phase space, the effects of nucleon absorption should be negligible. A zero-degree calorimeter or a forward hadron spectrometer, such as that of the BRAHMS detector at RHIC [76] could clarify this matter.

\section{Determining the importance of comovers.}

The use of onium production data for determining the nature of the QGP will be difficult if there are non-QGP mechanisms (other than simple shadow- 
ing) affecting onium production that are comparable to the predicted QGP effects. We have already discussed one such mechanism, namely nuclear absorption. However, we concluded that it was relatively benign since it should have little $p_{T}$ dependence and will not be important if the projectile and target fragmentation regions are well separated from the quarkonium states in the central region. A second mechanism of concern is the breakup/absorption of the onium states through interactions with comoving secondary hadrons. Indeed, the observed $J / \psi$ suppression [15, 16] at $\sqrt{s}=17 \mathrm{GeV}$ has been attributed to interactions with these comovers [20]. The comovers should also be present at higher energies. Such hadronic scattering can affect both the $\psi$ states and the $\Upsilon$ states. For example, the comovers can easily break up the $J / \psi$ if the combined comover $+J / \psi$ center-of-mass energy is above the $D \bar{D}$ threshold. Since the separation between the onium mass and the threshold is smaller for the higher onium states, the higher states will be more easily broken apart by comovers. Thus, comovers could cause ratios such as $\Upsilon^{\prime} / \Upsilon$ and $\psi^{\prime} / \psi$ to be smaller than expected. The $\Upsilon$, being the most tightly bound of the onium states, should be least affected. Note that this is precisely the same as expected from nucleon absorption, which should be largest for states with large radii and small mass thresholds. As discussed earlier, if the comovers are mesons, the onium-comover absorption cross section should be $\sim 2 / 3$ the onium-nucleon absorption cross section.

Although the interpretation of $J / \psi$ suppression at $\sqrt{s}=17 \mathrm{GeV}$ as being due to comover scattering implies high hadronic densities in the present experiments, a hadronic interpretation of quarkonium dissociation would be much less plausible at LHC energies, where, for all cases considered in Table 0 , $T_{0}$ is significantly above $T_{c}$. The reasoning is as follows. For such high initial temperatures, it is almost certain that a QGP will form, delaying the creation of hadrons until the $Q \bar{Q}$ pair or onium state has passed through much of the material. Thus, there would be little opportunity for interactions with comovers until the late stages of the collision. The $T_{0}$ values for RHIC (Table 6) are also generally higher than $T_{c}$ and significant breakup due to interactions with comovers again seems unlikely if a QGP is formed.

While the above argument suggests that comover absorption will not significantly impact onium production, it will be important to try to determine the importance of comovers directly from experiment. We outline several possible approaches.

- In $p A$ collisions a QGP is not formed. Thus, as noted earlier, in $p A$ collisions the dilepton spectrum in the $M>1 \mathrm{GeV}$ region, being primarily due to $c \bar{c}$ production and decay, should provide a reliable probe of shadowing. Comovers will have only a small influence on open charm mesons because the scattering is elastic - a $\pi D$ interaction will change the momentum distribution of the $D$ but will not take the charm out 
of the system. Nucleon- $D$ scattering is also expected to be elastic. On the other hand, a $J / \psi$-comover interaction is likely to break up the $J / \psi$ into $D \bar{D}$ pairs. By comparing the 'shadowing' on and off the resonance, we can obtain a firmer handle on the magnitude of comover absorption of the $J / \psi$.

- In nucleus-nucleus collisions, the density of comovers which can absorb the onium state would be larger still, with the largest density in central collisions. Since these densities are related to the final multiplicity and thus to the transverse energy of the collision, perhaps one of the best ways to check the importance of comover breakup and absorption is to trigger on peripheral collisions (for which a QGP is not formed). One would compare predictions for the $\psi$ and $\Upsilon$ states based on shadowing in peripheral collisions with actual measurements to see if there is extra suppression due to comover breakup or absorption. Such collisions could provide very valuable guidance on whether comover absorption is important. In addition, one could compare (as above) the dilepton spectrum on and off the $\psi$ resonance.

Comover absorption will also not occur if the onium $p_{T}$ is large enough. Calculations of comover suppression as a function of $p_{T}$ appear in Refs. [77, 78, 79. The range of $p_{T}$ over which comover suppression occurs should not depend strongly on energy. One can make a crude estimate of the $p_{T}$ range of comover interactions as in eq. (17). In this case,

$$
p_{T, \max }^{\mathrm{co}}=M \sqrt{\left(t_{\mathrm{co}} / \tau_{\mathrm{co}}\right)^{2}-1},
$$

where $t_{\text {co }}$ depends on the size of the system, $t_{\text {co }} \sim R / v_{\text {rel }}$, $\left(v_{\text {rel }}\right.$ is the relative velocity of the onium and the comovers, 0.6 for $\psi$-comover interactions at the CERN SPS) and $\tau_{\text {co }} \sim 2 \mathrm{fm}$ is the comover formation time in a nucleusnucleus collision. Since we assume comovers are final-state hadrons, there is no $T_{0}$ dependence and since the $p_{T}$ range depends on the comover formation time rather than the onium formation time, $p_{T, \max }^{\mathrm{co}}$ changes by $20 \%$ or less for each onium family. When $R=R_{\mathrm{Pb}}, p_{T \text {, } \operatorname{cox}}^{\mathrm{co}}=5.8 M$. Note that if a QGP is formed, $\tau_{\text {co }}$ could be even longer since the comovers would not form until after the QGP hadronizes. At the very least, the above comparisons should allow us to determine the maximum $p_{T}$ up to which comover absorption is important.

The influence of comover absorption is clearly an important issue. In the following discussion, we assume that all nuclear and comovers effects on the onium production cross sections can be unfolded or proven to be small.

\section{Using RHIC data to establish the general nature of the QGP.}


At RHIC ratios such as $\Upsilon^{\prime} / \Upsilon$ and $\psi^{\prime} / \psi$ will be measured in nucleusnucleus collisions. For minijet initial conditions or initial conditions based on the uncertainty relations, the short initial time scales result in the prediction that there should be no significant suppression at RHIC for any of the onium states, regardless of the form of $\mu(T)$. However, in the parton gas model the long $t_{0}$ implies that suppression of the $\Upsilon$ states is possible for the $\mathrm{SU}(N)$ (but not the 3-Flavor) form of $\mu(T)$. Further, the $\psi$ states are predicted to be suppressed for both the $\mathrm{SU}(N)$ and 3-Flavor forms of $\mu(T)$. Thus, the suppression pattern of the $\psi$ and $\Upsilon$ states will help distinguish the $\mu(T)$ dependence if significant suppression is observed. Specifically:

- If all the ratios are independent of $p_{T}$, then either no plasma has been produced or the initial conditions of the plasma are unfavorable for suppression to occur.

- If the $\psi^{\prime} / \psi$ ratio depends on $p_{T}$, but the $\Upsilon^{\prime} / \Upsilon$ ratio does not, the most consistent picture is that a QGP has formed with initial conditions like those of the parton gas model and $\mu(T)$ is of the 3-Flavor form given in eq. (6).

- If both ratios are $p_{T}$-dependent, then parton-gas-like initial conditions are required and the screening mass is more likely to be of the $\mathrm{SU}(N)$ form as in eq. (5).

\section{Analyzing the QGP at the LHC.}

One advantage of the LHC over RHIC is that onium suppression can occur for a much broader range of possible initial conditions. In particular, at the LHC the minijet initial conditions produce an initial temperature $T_{0}$ that is sufficiently large that the fast equilibration predicted (i.e. small $t_{0}$ ) does not prevent suppression. More generally, the higher $T_{0}$ possible at the LHC will be crucial since fast equilibration is perhaps more plausible.

The question we now address is how to best determine $t_{0}$ and other features of the QGP. A high statistics study of the $\Upsilon^{\prime} / \Upsilon$ ratio as a function of $p_{T}$ may prove conclusive. If significant $p_{T}$-dependence of $\Upsilon^{\prime} / \Upsilon$ is found for $p_{T} \gtrsim 10 \mathrm{GeV}$ in $\mathrm{Pb}+\mathrm{Pb}$ collisions at $5.5 \mathrm{TeV}$, then it will be virtually certain that a quark gluon plasma was formed. (As argued at the end of the previous section, any $p_{T}$-dependence of this type of ratio arising from low- $p_{T}$ perturbative or non-perturbative resummation effects should be small, and effects from comovers/absorption/shadowing should be absent once $p_{T}$ is reasonably large.) The precise behavior of the $\Upsilon^{\prime} / \Upsilon$ ratio can then be used to strongly constrain the QGP model parameters. In particular, the ratio will be very different if only the $\Upsilon^{\prime}$ is suppressed in comparison to the case where both states are suppressed. 


\section{Prompt Production Ratios}

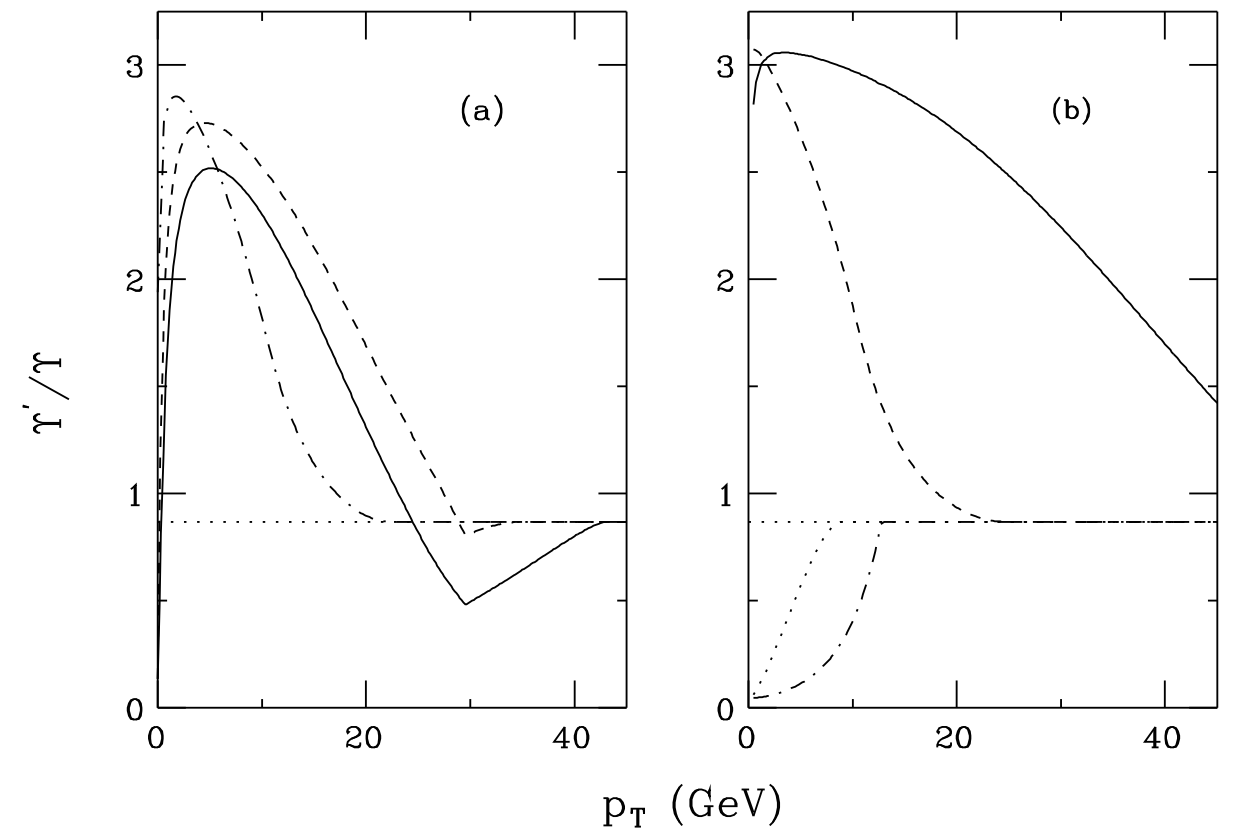

Figure 6: The direct or prompt $\Upsilon^{\prime} / \Upsilon$ ratio as a function of $p_{T}$ is shown for several choices of initial conditions at the LHC. In (a), we show results for the minijet model with the $\mathrm{SU}(N)$ form of $\mu(T)$, eq. (5), (for which $T_{0}=1.14 \mathrm{GeV}$ and $t_{0}=0.1 \mathrm{fm}$ ) taking $R=R_{\mathrm{Pb}}$ (solid), $R=R_{\mathrm{S}}$ (dashed), $R=1 \mathrm{fm}$ (dot-dashed). In (b), we consider the parton gas model with $T_{0}=0.82 \mathrm{GeV}$ and $t_{0}=0.5 \mathrm{fm}$, and show results for the $\mathrm{SU}(N) \mu(T)$ with $R=R_{\mathrm{Pb}}$ (solid) and $R=1 \mathrm{fm}$ and for the 3-Flavor $\mu(T)$ from eq. (6) with $R=R_{\mathrm{Pb}}$ (dot-dashed) and $R=1 \mathrm{fm}$ (dotted).

In Fig. 6, we show the $\Upsilon^{\prime} / \Upsilon$ ratio for several sets of initial conditions. The 'prompt' ratio displayed is that obtained if only directly produced $\Upsilon^{\prime}$ and $\Upsilon$ states are included. In other words, the ratio is computed using $f_{\Upsilon^{\prime}}^{d}$ and $f_{\Upsilon}^{d}$ from eq. (13). We will return shortly to the issue of whether or not this prompt ratio can be experimentally measured.

Fig. 6(a) displays results obtained when the minijet initial conditions are used with $t_{0}=0.1 \mathrm{fm}$ and $\mu(T)$ is as given in eq. (5). Both the $\Upsilon$ and $\Upsilon^{\prime}$ states are suppressed and the solid, dashed and dot-dashed curves (for $R=R_{\mathrm{Pb}}, R_{\mathrm{S}}$ and $1 \mathrm{fm}$, respectively) show that the $p_{T}$-dependence of the ratio can distinguish different plasma radii. At low $p_{T}$ the $\Upsilon$ is actually more suppressed than the $\Upsilon^{\prime}$, leading to a significantly larger ratio than that expected in low energy $p p$ collisions (or found in current $p \bar{p}$ measurements). For a large $R$, the ratio eventually drops below the value expected in the 
absence of QGP effects (i.e. for large enough $p_{T}$ ). The point at which this shift occurs is at $p_{T} \approx 25 \mathrm{GeV}$ if $R=R_{\mathrm{Pb}}$ and at $\approx 29 \mathrm{GeV}$ for $R=R_{\mathrm{S}}$. The kink in these ratios at $30 \mathrm{GeV}$ occurs at the point where the $\Upsilon$ is no longer suppressed. In a system with a smaller $R=1 \mathrm{fm}$ radius, the $\Upsilon$ is always more suppressed than the $\Upsilon^{\prime}$ so that the ratio returns to the $p p$ value at $p_{T} \approx 20 \mathrm{GeV}$.

Fig. 6(b) shows the ratio computed using the parton gas initial conditions. When the $\mathrm{SU}(N) \mu(T)$ is assumed, the behavior is similar to the minijet case although when $R=R_{\mathrm{Pb}}$ the increase in $\Upsilon^{\prime} / \Upsilon$ persists to much larger $p_{T}$. When $R=1 \mathrm{fm}$, the result is almost indistinguishable from the minijet case with the same radius. The maximum of the ratio is $\sim 10 \%$ larger at $p_{T} \sim 1-2$ $\mathrm{GeV}$, but this may be difficult to distinguish. If the 3-Flavor [eq. (6)] scenario is assumed for the screening mass, then the $\Upsilon^{\prime}$ is suppressed while the $\Upsilon$ is not and the $p_{T}$-dependence of the ratio is quite different. The dot-dashed and dotted curves of Fig. 6(b) show that until the effects of the plasma disappear, the ratio is less than expected in the absence of QGP formation. However, it is more difficult to differentiate between a system the size of the lead nucleus or a system with $R=1 \mathrm{fm}$ unless the measurement is made with high statistics. We have already noted that if significant suppression is observed at RHIC in the $\psi^{\prime} / \psi$ ratio as a function of $p_{T}$ and $\Upsilon$ suppression is observed at RHIC, the general behavior of $\mu(T)$ should be relatively clear by the time the LHC measurement is made.

Finally, we recall that for the uncertainty relation initial conditions, both $t_{0}$ and $T_{0}$ are small even at the LHC and no suppression is expected for the Upsilon states. To learn about the existence and nature of a QGP via measurements of onium production will not be possible for such models.

\section{The importance of separating prompt from indirectly produced onium states.}

An important issue is whether or not the 'prompt' ratio discussed above can be extracted experimentally. We saw earlier that a significant fraction of $\Upsilon$ production arises from $\Upsilon^{\prime}$ and $\chi_{b}\left(\chi_{b}\right.$ denotes the collective $\chi_{b i}(1 P, 2 P), i=$ $0,1,2$ states) production followed by $\Upsilon^{\prime} \rightarrow \Upsilon X$ and $\chi_{b} \rightarrow \gamma \Upsilon$, respectively. Further, some of the $\Upsilon^{\prime}$ 's come from $\chi_{b i}(2 P) \rightarrow \gamma \Upsilon^{\prime}$. We can only isolate the prompt ratio discussed above if we can veto such decay sources of the $\Upsilon$ and $\Upsilon^{\prime}$. In the case of the $\chi_{b}$ decays, this will only be possible by detecting the associated photon and reconstructing the $\chi_{b}$. The ability of the LHC detectors to do this will be limited at low $p_{T}$ for two reasons: i) there is a large $\pi^{0}$ background from which it is difficult to extract isolated $\gamma^{\prime}$ 's until the $p_{T}$ of the $\gamma$ becomes substantial $(\gtrsim 10 \mathrm{GeV})$ and ii) the photon energy resolution will be poor at low $p_{T}$. In order to veto $\Upsilon^{\prime} \rightarrow \Upsilon X$ decays we must be able to detect the $X=\pi^{+} \pi^{-}, \pi^{0} \pi^{0}$ decay products. This latter may be impossible; however, this indirect decay is only responsible for about $13 \%$ of 


\section{Production Ratios With Indirect Decays}

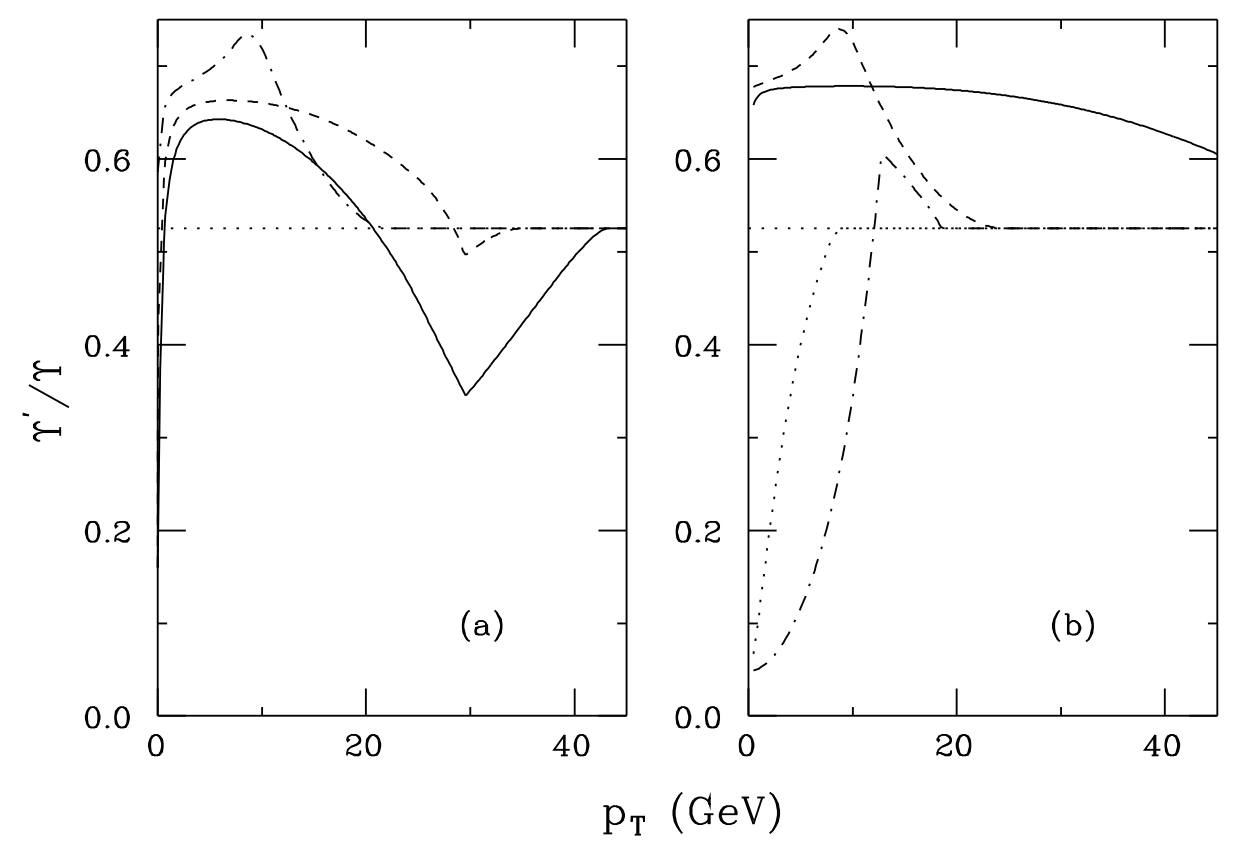

Figure 7: The $\Upsilon^{\prime} / \Upsilon$ ratio including indirect decays as a function of $p_{T}$ is shown for several choices of initial conditions at the LHC. In (a), we show results for the minijet model with the $\mathrm{SU}(N)$ form of $\mu(T)$, eq. (5), (for which $T_{0}=1.14 \mathrm{GeV}$ and $t_{0}=0.1 \mathrm{fm}$ ) taking $R=R_{\mathrm{Pb}}$ (solid), $R=R_{\mathrm{S}}$ (dashed), $R=1 \mathrm{fm}$ (dot-dashed). In (b), we consider the parton gas model with $T_{0}=0.82 \mathrm{GeV}$ and $t_{0}=0.5 \mathrm{fm}$, and show results for the $\mathrm{SU}(N) \mu(T)$ with $R=R_{\mathrm{Pb}}$ (solid) and $R=1 \mathrm{fm}$ and for the 3-Flavor $\mu(T)$ from eq. (6) with $R=R_{\mathrm{Pb}}$ (dot-dashed) and $R=1 \mathrm{fm}$ (dotted).

the $\Upsilon^{\prime}$ 's, so that if the $\chi_{b}$ decays can be vetoed we will obtain a result much like the prompt ratio plotted in Fig. 6. If the $\chi_{b}$ decays cannot be vetoed, then we must consider all sources of the $\Upsilon^{\prime}$ and $\Upsilon$, each of which will be associated with a different suppression factor. Numerically, it is sufficient to consider:

$$
\frac{\Upsilon^{\prime}+\chi_{b}(2 P)\left(\rightarrow \Upsilon^{\prime}\right)+\Upsilon^{\prime \prime}\left(\rightarrow \Upsilon^{\prime}\right)}{\Upsilon+\chi_{b}(1 P, 2 P)(\rightarrow \Upsilon)+\Upsilon^{\prime}(\rightarrow \Upsilon)+\Upsilon^{\prime \prime}(\rightarrow \Upsilon)}
$$

In computing this 'indirect' $\Upsilon^{\prime} / \Upsilon$ ratio we have assumed that the survival probability, $S\left(p_{T}\right)$, of the $\chi_{b}(2 P)$ states is the same as that for the $\chi_{b}(1 P)$ states and that $S\left(p_{T}\right)$ for the $\Upsilon^{\prime \prime}$ is the same as for the $\Upsilon^{\prime}$.

The indirect ratio, defined in eq. (25) is plotted in Fig. 7. Clearly, the indirect ratio is somewhat less sensitive to changes in $R$ and $\mu(T)$ than is the direct $\Upsilon^{\prime} / \Upsilon$ ratio. If the $\mathrm{SU}(N)$ form of $\mu(T)$ is appropriate, the figure indi- 
cates that a measurement at the $20 \%$ level is needed to distinguish between the $p p$ value of the ratio and the QGP prediction including indirect decays in both the minijet and parton gas initial condition scenarios. (If there are substantial systematic errors in the ratio, the detection of a deviation might be most difficult for the parton gas $\mathrm{SU}(N)$ case when $R=R_{\mathrm{Pb}}$, since in this case the ratio varies slowly with $p_{T}$.) If the slowly-growing 3-Flavor form of $\mu(T)$ is correct, the dot-dash and dotted curves of Fig. 7(b) show that the parton gas model predicts significant and strongly $p_{T}$-dependent departures from the $\Upsilon^{\prime} / \Upsilon$ ratio expected in the absence of a QGP. It would even be possible to determine the size of the system using the indirect ratio - as shown in Fig. 7(b) the $\chi_{b}$ contribution to the $\Upsilon$ causes the $\Upsilon^{\prime} / \Upsilon$ ratio to increase above the $p p$ level at $p_{T} \approx 12 \mathrm{GeV}$ for $R=R_{\mathrm{Pb}}$ but not for $R=1$ fm.

Thus, all is not lost if only the indirect ratio can be measured, but sensitivity is reduced and the accuracy with which the $\Upsilon^{\prime} / \Upsilon$ ratio can be measured becomes crucial. Also, our ability to predict theoretically or measure in other contexts with some reliability the relative values of the $f^{d}$ 's becomes quite important.

\section{Estimates of the statistics required and the importance of vetoing indirect decays.}

The ability to employ the $\Upsilon^{\prime} / \Upsilon$ ratio to analyze the nature of the QGP will depend crucially on the statistics available. For $\sigma_{N N} T_{\mathrm{PbPb}}(\mathbf{0}) L_{\mathrm{int}}^{\mathrm{PbPb}}=$ $4.973 \times 10^{3} \mathrm{nb}^{-1}$ per month, as assumed in Table 4 , and the $d \sigma(p p) / d p_{T} \times$ $S\left(p_{T}\right)$ spectra of Fig. 5, which range very roughly from $1 \mathrm{nb} / \mathrm{GeV}$ to 0.1 $\mathrm{nb} / \mathrm{GeV}$ over the $p_{T}$ range of interest, we find $\sim 5 \times 10^{3}$ falling to $\sim 5 \times 10^{2}$ events per $\mathrm{GeV}$ for the individual $\Upsilon, \Upsilon^{\prime}$ and $\chi_{b}$ resonances. For branching ratios of $B_{\Upsilon}=0.025$ and $B_{\Upsilon^{\prime}}=0.013$ and an efficiency/acceptance factor of $33 \%$ (which, as noted earlier, applies to the CMS detector), this leaves us with $\sim 41.3(\sim 21.5)$ falling to $\sim 4.13(\sim 2.15) \Upsilon\left(\Upsilon^{\prime}\right)$ events per $\mathrm{GeV}$ per month of running. For a $2 \mathrm{GeV}$ bin size, 12 months of running and negligible background (see below), we obtain $\Upsilon\left(\Upsilon^{\prime}\right)$ statistical accuracies of $\sim 3 \%(\sim 4.5 \%)$ at low $p_{T}$ rising to $\sim 10 \%(\sim 14 \%)$ at $p_{T} \sim 25-30$ for the individual spectra, and of $5.4 \%$ rising to $17 \%$ for the $\Upsilon^{\prime} / \Upsilon$ ratio. Thus, if the prompt ratio can be extracted, somewhat more than a year of running at $L_{\mathrm{int}}^{\mathrm{PbPb}}=2.59 \mathrm{nb}^{-1}$ per month would be sufficient to measure the ratios with the required accuracy over the full range of $p_{T}$ values of interest. If the $\Upsilon^{\prime} / \Upsilon$ ratio can only be measured including indirect decays, greater accuracy is required at large $p_{T}$. Roughly 36 months of running at $L_{\mathrm{int}}^{\mathrm{PbPb}}=2.59 \mathrm{nb}^{-1}$ per month would be required to achieve $\lesssim 10 \%$ accuracy for the ratio at $p_{T} \sim 30 \mathrm{GeV}$. Since this amount of running in the heavy ion mode at the LHC is quite unlikely, we see how important the vetoing of indirect decays is if we are to use the $\Upsilon^{\prime} / \Upsilon$ ratio to analyze the QGP. 


\section{The importance of mass resolution.}

Also crucial to the program is the ability to resolve the $\Upsilon, \Upsilon^{\prime}$ and $\Upsilon^{\prime \prime}$ resonances from one another. The CMS detector provides excellent mass resolution in the $\mu^{+} \mu^{-}$mode, of order $40 \mathrm{MeV}$, adequate for clean separation of the $\Upsilon, \Upsilon^{\prime}$ and $\Upsilon^{\prime \prime}$ signals (see Fig. 12.31b in Ref. [21]). As also shown in [21], the excellent resolution implies a very modest level of background under the $\Upsilon$ peaks. Preliminary estimates of the mass resolution for the ATLAS detector are less encouraging, of order $200-300 \mathrm{MeV}$ [80], for which separation of the resonances would not be possible.

\section{Other onium ratios.}

In the above discussion, we have focused on the $\Upsilon^{\prime} / \Upsilon$ ratio. Other ratios would provide complementary information. However, the $\Upsilon^{\prime \prime} / \Upsilon$ ratio is a factor of 4 smaller [see eq. (13)] at the direct, i.e. prompt, level and statistics would be a problem. The direct/prompt $\chi_{b}(1 P) / \Upsilon$ ratio is similar to the $\Upsilon^{\prime} / \Upsilon$ ratio, the major difference being that the $\chi_{b}(1 P) / \Upsilon$ ratio never decreases below the $p p$ level at high $p_{T}$ in the $\mathrm{SU}(N)$ case since $p_{T, \text { max }}^{\chi_{b}} \approx p_{T, \text { max }}^{\Upsilon}$ (see Figs. 橉 and (1). Further, if the $\chi_{b}(1 P)$ radiative decays can be detected with reasonable efficiency, the statistical accuracy with which the $\chi_{b}(1 P) / \Upsilon$ ratio can be measured would be comparable to that for the $\Upsilon^{\prime} / \Upsilon$ ratio, thus making it a most valuable addition. This adds to the importance of being able to trigger efficiently on the decay photons so as to isolate these two direct ratios.

\section{Extracting individual survival probabilities and using $Z$ production to control systematics.}

So far, we have considered only ratios of onium rates. However, it will be important to know that suppression of the general type expected is taking place in the individual spectra contributing to these ratios. It would be even more valuable if a quantitative determination of $S\left(p_{T}\right)$ for the different resonances were possible through a direct comparison of the differences between the $p p$ spectra and the $\mathrm{Pb}+\mathrm{Pb}$ spectra at $5.5 \mathrm{TeV}$, as illustrated in Fig. 5 . In principle, the ratio of the properly normalized $\mathrm{Pb}+\mathrm{Pb}$ spectrum to the $p p$ spectrum for a given resonance simply gives $S\left(p_{T}\right)$, 1 provided there are no non-QGP effects that can also alter the $\mathrm{Pb}+\mathrm{Pb}$ spectrum. However, it is very likely that there will be some nuclear effects from shadowing, comovers and absorption. Effects from comovers and absorption cannot be disentangled except as described earlier. However, there is some possibility of obtaining additional information regarding shadowing.

The strength of shadowing depends upon the $x$ values at which the parton distributions are probed. Thus, the RHIC $p A$ measurements of onia produc-

\footnotetext{
${ }^{11}$ As argued in the previous section, non-perturbative resummation effects upon the $p_{T}$ distributions will cancel out in such a ratio.
} 
tion, while helpful, cannot directly determine the full effect of shadowing at $5.5 \mathrm{TeV}$ where we wish to determine the presence of the QGP and measure $S\left(p_{T}\right)$. An independent test of the shadowing effect using measurements at $\sqrt{s}=5.5 \mathrm{TeV}$ would be of great value for checking our understanding of shadowing and assessing the accuracy with which it can be unfolded from the data so as to reveal the QGP effects directly.

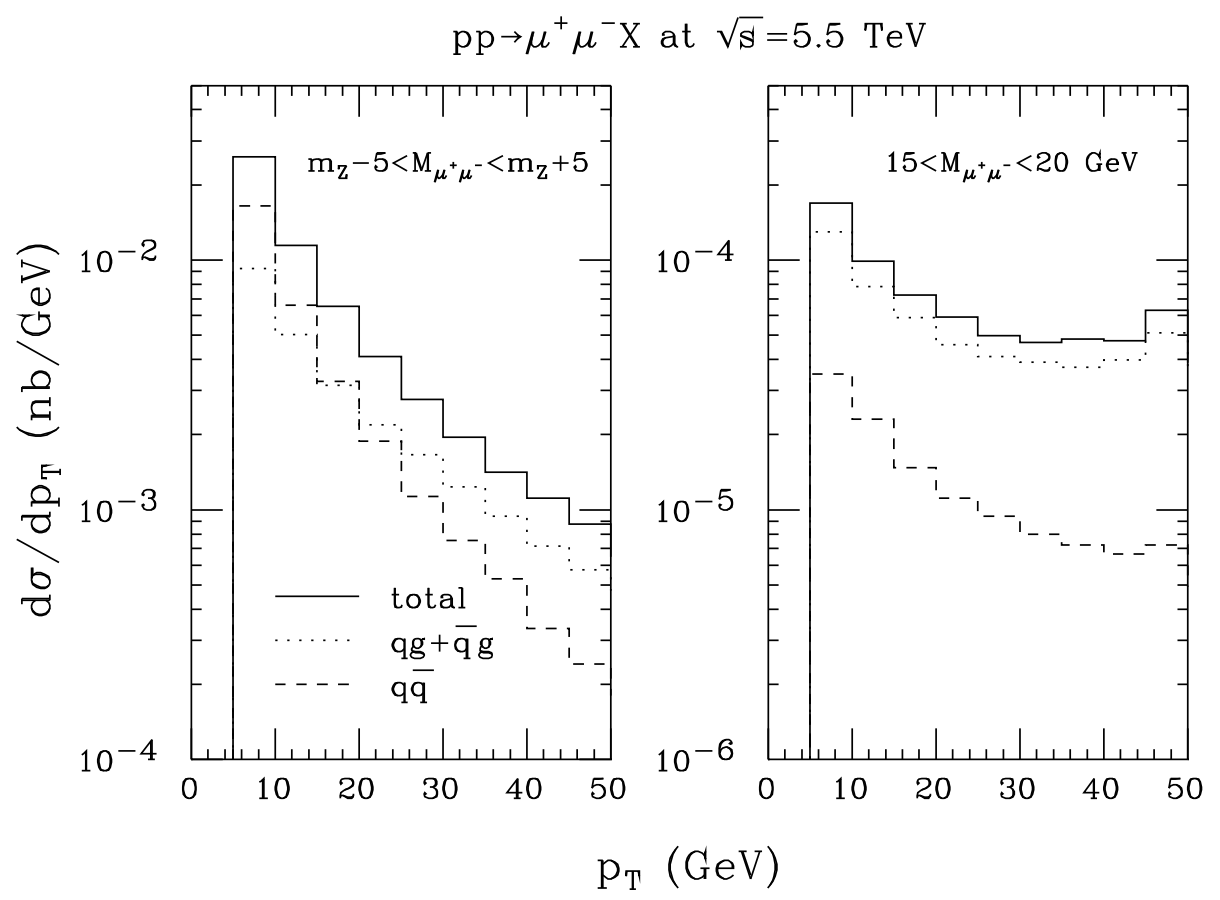

Figure 8: In (a), we plot $d \sigma / d p_{T}$ for $p p \rightarrow \mu^{+} \mu^{-} X$ at $\sqrt{s}=5.5 \mathrm{TeV}$ as a function of $p_{T}$ for $Z$ production (defined by $m_{Z}-5 \mathrm{GeV} \leq M_{\mu^{+} \mu^{-}} \leq m_{Z}+$ $5 \mathrm{GeV}$ ). In (b), the same cross section is plotted for $15 \mathrm{GeV} \leq M_{\mu^{+} \mu^{-}} \leq$ $20 \mathrm{GeV}$. The separate contributions from $q \bar{q}$ and $q g+\bar{q} g$ collisions are indicated by dashed and dotted histograms, respectively.

In the current experiments at the CERN SPS, $J / \psi$ production is compared to the dilepton continuum [15, 16]. The continuum is assumed to be produced via the Drell-Yan $\left(\gamma^{\star}, Z^{\star}\right.$-exchange) process and is, in fact, DrellYan-like in its behavior. At RHIC energies and higher, the continuum will be more difficult to understand because of the important contribution from semileptonic $c \bar{c}$ and $b \bar{b}$ decays. Not only are there uncertainties in the total $c \bar{c}$ cross section, but the heavy quark decays are also subject to nuclear effects. The relatively small Drell-Yan contribution is also subject to shadowing effects in the mass range between the $J / \psi$ and the $\Upsilon$. Another choice is needed. One possibility is $Z$ production. Because the $Z$ is produced in 
point-like fashion, the difference between the $p_{T^{-}}$-dependence of the $Z$ in $p p$ collisions vs. $\mathrm{Pb}+\mathrm{Pb}$ collisions will not be influenced by the quark-gluon plasma $[2$.

Figure 8 illustrates the cross section for $Z$ production as a function of $p_{T}$ at $\sqrt{s}=5.5 \mathrm{TeV}$ assuming no shadowing. There, we plot $d \sigma / d p_{T}$ for $p p \rightarrow Z X$ and the separate contributions from $q \bar{q}$ and $q g+\bar{q} g$ collisions. (The distributions include 1-loop corrections to the $Z+$ jet cross section[ $[$ ], but do not include low- $p_{T}$ resummation effects.) To obtain the number of events per month per $\mathrm{GeV}$ coming from central collisions ${ }^{\text {Tt }}$, we multiply by the factor $\sigma_{N N} T_{\mathrm{PbPb}}(\mathbf{0}) L_{\mathrm{int}}^{\mathrm{PbPb}}=4.973 \times 10^{3} \mathrm{nb}^{-1}$. At $p_{T}=50 \mathrm{GeV}$, the cross section is of order $10^{-3} \mathrm{nb} / \mathrm{GeV}$, implying about 2 events per $\mathrm{GeV}$ for $40 \%$ acceptance and detection efficiency. Thus, for a $5 \mathrm{GeV}$ bin we obtain about 10 events in this bin per month of running. After a year of running, this would yield a statistical accuracy of order $9 \%$. At low $p_{T}$, event rates are a factor of $\gtrsim 10$ larger, yielding correspondingly greater accuracy. The predicted effects of the QGP, see for example Figs. 3 and 4 , typically imply survival probabilities that differ by much larger percentages compared to unity. In any case, as estimated earlier, the errors in the measurements of the Upsilon resonance spectra will be larger. Thus, production rates in the $p_{T} \lesssim 50 \mathrm{GeV}$ domain are high enough that $Z \rightarrow e^{+} e^{-}, \mu^{+} \mu^{-}$can provide a standard of comparison. Of course, we will also wish to measure $Z$ production in $p p$ collisions at $\sqrt{s}=5.5 \mathrm{TeV}$ so as to determine if shadowing and other nuclear effects have impacted the $\mathrm{Pb}+\mathrm{Pb}$ spectra. Good statistical accuracy for up to $p_{T}=50 \mathrm{GeV}$ requires integrated luminosity for $p p$ collisions of order $L=0.01 \mathrm{fb}^{-1}$, which should be easily achieved, even at this reduced energy, by running for a few weeks.

The two difficulties with using $Z$ production as a benchmark are: (i) $m_{Z}$ is much larger than the masses of the Upsilon resonances and (ii) the $q \bar{q}$ and $q g+\bar{q} g$ parton luminosities are probed vs. the $g g$ luminosities responsible for $b \bar{b}$ production.

- The large size of $m_{Z}$ compared to the Upsilon family masses reduces the value of $Z$ production as a benchmark for two reasons. First, shadowing and related nuclear effects may well be dependent upon $Q^{2}$. For

\footnotetext{
${ }^{12}$ Once again, this ratio will be very insensitive to low- $p_{T}$ perturbative and nonperturbative resummation modeling.

${ }^{13}$ We thank U. Baur for providing a program against which to check our own calculations.

${ }^{14} \mathrm{We}$ wish to restrict observations to central collisions in case shadowing and nuclear effects (that might possibly be present for the $Z$ ) for peripheral collisions are somewhat different.

${ }^{15}$ If we assume that low-luminosity running yields $p p$ integrated luminosity of $10 \mathrm{fb}^{-1}$ per year at $\sqrt{s}=14 \mathrm{TeV}$ and that instantaneous luminosity falls as $E^{2}$, then the corresponding integrated luminosity at $\sqrt{s}=5.5 \mathrm{TeV}$ would be $\sim 1.5 \mathrm{fb}^{-1}$ per year or $\sim 0.03 \mathrm{fb}^{-1}$ per week.
} 
example, in the limit of large $Q^{2}$, holding the parton $x$ fixed, shadowing becomes stronger in some models (see, e.g. , Ref. [81] whereas in others it is predicted to disappear altogether (see, e.g. , Ref. 82]). See also Ref. 83. Thus, it is very possible that the shadowing at $Q^{2}=M_{\Upsilon}^{2}$ will differ substantially from that at $Q^{2}=m_{Z}^{2}$. Second, the $x$ values probed $\left(x \sim m_{Z} / \sqrt{s} \sim 0.016\right.$ at $\left.y=0\right)$ are much larger than in Upsilon production at the same energy. (Detailed measurements as a function of the $Z$ rapidity would provide information about smaller $x$ values, but require more luminosity for good statistics.)

- The separate contributions of the $q \bar{q}$ vs. $q g+\bar{q} g$ densities to the $Z$ spectrum appear in Fig. 8. We observe that $q \bar{q}$ collisions are dominant for $p_{T} \lesssim 15 \mathrm{GeV}$; for higher $p_{T}$ values $q g+\bar{q} g$ collisions dominate. Thus, to probe nuclear effects on the $g$ distribution at $Q^{2}=m_{Z}^{2}$, we must have knowledge of their effects on the $q$ and $\bar{q}$ distributions for $x$ values in the vicinity of those being probed at this same $Q^{2}$. At low to moderate $Q^{2}$ the nuclear effects on $q$ and $\bar{q}$ PDFs are rather well measured and do not appear to be strongly $Q^{2}$ dependent [57]. However, there is currently no direct measurement of $q$ and $\bar{q}$ shadowing at small $x$ with $Q^{2}$ values as high as $m_{Z}^{2}$. If nuclear beams become available at HERA, then direct measurement of shadowing at appropriate $x$ and $Q^{2}$ values would be possible.

Even though $Z$ production does not directly probe gluon shadowing at the $x$ and $Q^{2}$ values of interest for Upsilon production, $Z$ production would be of considerable value in developing a reasonable theoretical understanding or model of shadowing. If we measure the $p_{T}$ dependence of $Z$ production and find that it is consistent with one or more theoretically reasonable models of shadowing, this will already be a very important benchmark in that we can be confident there is not some totally unexpected physics occurring in nucleonnucleon collisions. Of course, it is vital that $Z$ production be measured both in $\mathrm{Pb}+\mathrm{Pb}$ and in $p p$ collisions at $\sqrt{s}=5.5 \mathrm{TeV}$ in order for us to develop confidence that we understand the impact of shadowing and other purely nuclear effects.

Given the above issues regarding $Z$ production, it would be advantageous if we could measure lepton pair production at pair masses nearer to those of the Upsilon states so as to constrain shadowing and nuclear effects at parton $x$ and $Q^{2}$ values closer to those of direct relevance. We have already noted that we expect a large background from $c \bar{c}$ and $b \bar{b}$ production processes for lepton pair masses lower than the Upsilon family masses. At these low masses, this background will be very difficult to veto by requiring that the leptons be isolated because of the high density of soft tracks in the $\mathrm{Pb}+\mathrm{Pb}$ collision environment. In the mass region above about $15 \mathrm{GeV}$ the dilepton rate from 
$c \bar{c}(b \bar{b})$ pair production is predicted to be smaller than (comparable to) that from $\gamma^{\star}, Z^{\star}$-exchange [84]. Further, in this higher mass range, vetoing the $b \bar{b}$ component using isolation requirements on the leptons might prove feasible at a level adequate to extract the pure DY dilepton spectrum. In Fig. 8, we have plotted $d \sigma / d p_{T}$ for production of muon pairs with $15 \mathrm{GeV} \leq M_{\mu^{+} \mu^{-}} \leq$ $20 \mathrm{GeV}$ coming from $\gamma^{\star}, Z^{\star}$-exchange. We note that in this case the $q g+\bar{q} g$ collision component is always dominant, as would be desirable for learning as much about gluon shadowing as possible. However, the cross section is nearly a factor of 100 below that for production at the $Z$ resonance, implying that statistics would be a factor of 10 worse. Even a year of running will not provide enough $\mathrm{Pb}+\mathrm{Pb}$ luminosity to yield measurements that are sufficiently accurate to constrain the shadowing and nuclear effects at the needed level ( $\lesssim 5-10 \%$ ). Thus, the low rate and uncertainty regarding our ability to veto the $b \bar{b}$ background imply that we should not count on being able to use dilepton pairs below the $Z$ mass region to improve our understanding of nuclear effects on the gluon PDFs. Nonetheless, the possibility of doing so should not be ignored and appropriate data, including event characteristics that might allow vetoing, should be collected.

HERA measurements of the nuclear $g, q$, and $\bar{q}$ densities as a function of $x$ and $Q^{2}$ would greatly assist in a reliable extraction of $S\left(p_{T}\right)$. Measurements overlapping the regions of interest for $\Upsilon$ and $Z$ production at $5.5 \mathrm{TeV}$ would be possible for an appropriate design of the facility. Using the HERA measurements for $x \sim 0.02$ and $Q^{2}=m_{Z}^{2}$, it would be possible to unfold the shadowing effects in $Z$ production and verify that the underlying $p_{T}$ spectrum is that expected on the basis of the Drell-Yan mechanism. This would allow a high degree of confidence that we could use the HERA measurements at the $x$ and $Q^{2}$ values appropriate to onium production to unfold the underlying $p_{T}$ spectra of the onia as influenced only by absorption, comovers, and the quark-gluon plasma, with the latter hopefully having a much larger impact.

\section{Discussion and conclusions}

We have found that expectations for $\Upsilon$ (and also $\psi$ ) resonance production in $\mathrm{Pb}+\mathrm{Pb}$ collisions at the LHC are very dependent upon the nature and details of the quark-gluon plasma. We have demonstrated that, far from being an impediment to our extracting the QGP physics, this dependence may very well allow us to determine much about the fundamental nature of the QGP, including: the energy density, the initial temperature, the plasma radius, and the temperature-dependent screening mass. Although we have kept the discussion of quarkonium production and suppression as general as possible, we chose the $\Upsilon$ family to examine in detail. The $\Upsilon$ rate is high 
enough for statistically significant measurements to be made, particularly at the LHC. The $\Upsilon$ is also less affected by interactions with hadronic matter, providing a less ambiguous signal than the charmonium states. Strikingly different expectations for the $p_{T}$-dependence of the $\Upsilon^{\prime} / \Upsilon$ ratio, the example upon which we focused, are found depending upon whether or not the QGP is formed and on the QGP properties. While our calculations of the survival probability are rather schematic, e.g. assuming full equilibration, they reflect the correct general trend for the scenarios discussed.

The much higher energy of the LHC could be crucial for QGP suppression of onium production to be possible. This would be the case, for example, in the minijet model of the initial conditions. The high minijet density at the LHC implies that the $p_{T}$ spectra of the $\Upsilon$ resonances in this model are likely to be highly sensitive to details of QGP formation. In contrast, at RHIC, the minijet density will be a factor of 10 to 30 smaller, and the maximum initial temperature predicted is $T_{0} \sim 0.5 \mathrm{GeV}$. Thus no suppression is expected. Initial conditions such as those predicted in parton gas models, for which the QGP takes longer to equilibrate, predict still stronger suppression at the LHC and could also lead to some suppression being observed at RHIC, depending upon the temperature dependence of the screening mass in the $b \bar{b}$ potential. There are also models of the initial conditions that predict no suppression even at the LHC. We point out that even a very high $T_{0}$ does not guarantee plasma suppression. Models assuming a slow equilibration result in greater suppression because of the longer time the system spends in the screening region with $T>T_{D}$.

Even if suppression is not present at RHIC, it is only at RHIC that $p A$ measurements will be possible. Despite the lower energy, these $p A$ measurements will greatly aid in constraining and checking our understanding of nuclear effects, such as those associated with shadowing, comover interactions and nuclear absorption. Thus, RHIC measurements will, as a minimum, provide important benchmarks.

We have demonstrated that statistics should be adequate to detect the differences in the $\Upsilon^{\prime} / \Upsilon$ ratio that would discriminate between different QGP models (assuming that the ratio can be measured at the prompt level). Although this (and other) ratios have the advantage that various systematic effects, as well as the purely nuclear effects associated with shadowing, will cancel, further vital information can be extracted if the $p_{T}$ spectra for the individual resonances can be measured and the effects of ordinary nuclear shadowing on these spectra unfolded. We have pointed to a comparison with $Z$ production as a potentially useful benchmark in this unfolding process. The ideal situation arises if, in addition to LHC $Z$ data, HERA nuclear data on shadowing is available for the $x$ and $Q^{2}$ values appropriate for both onium and $Z$ production at the LHC. The observed $p_{T}$ spectrum for the $Z$ could be 
compared to the prediction based on the Drell-Yan process using the experimentally measured nuclear PDFs from HERA. If there is agreement, then we would have a high level of confidence that we can unfold the shadowing effects on the onia $p_{T}$ spectra, using the PDFs determined from HERA data at the appropriate $x$ and $Q^{2}$ values, so as to expose the impact of the QGP (which hopefully will be dominant over absorption and comovers). Whether or not the HERA data is available, $Z$ production will provide an important benchmark as to how much nuclear shadowing effects alone are likely to be impacting the measured $p_{T}$ spectra of the onia.

Certain detector features will play a key role in carrying out the analysis as envisioned here. Of greatest importance is the mass resolution in the $\mu^{+} \mu^{-}$ channel. The resolution for the CMS detector, as quoted in their technical proposal, is such that the $\Upsilon, \Upsilon^{\prime}$ and $\Upsilon^{\prime \prime}$ resonances can be cleanly separated from one another with very modest background under the peaks. Less certain is the extent to which the $\chi_{b} \rightarrow \gamma \Upsilon$ process can be separated from direct $\Upsilon$ production by detecting the associated $\gamma$ for $p_{T}$ 's in the range of interest. The statistics necessary to discriminate between different QGP models is much greater if the direct 'prompt' $\Upsilon^{\prime} / \Upsilon$ ratio cannot be isolated. One also would lose the ability to observe the $\chi_{b} / \Upsilon$ ratio, which exhibits sensitivity to the QGP model similar to $\Upsilon^{\prime} / \Upsilon$, thus roughly doubling the significance with which different QGP models can be differentiated. It will be important for the LHC detector groups to give further attention to both experimental issues.

\section{Acknowledgements}

We would like to thank S. Brodsky, D. Denegri, W. Geist, W. Ko and P. Levai for discussions. We thank U. Baur for providing a $Z$ production program against which to check our results. R.V. thanks the Institute for Nuclear Theory at the University of Washington for its hospitality during the completion of this work. 


\section{References}

[1] F. Karsch, Nucl. Phys. A590 (1995) 367c.

[2] F.R. Brown et al., Phys. Rev. Lett. 65 (1990) 2491.

[3] T.K. Gaisser and F. Halzen, Phys. Rev. Lett. 54 (1985) 1754; L. Durand and H. Pi, Phys. Rev. Lett. 58 (1987) 303; G. Pancheri and Y.N. Srivastava, Phys. Lett. B182 (1986) 199.

[4] C. Albajar et al., UA1 Collab., Nucl. Phys. B309 (1988) 405.

[5] K.J. Eskola and M. Gyulassy, Phys. Rev. C47 (1993) 2329.

[6] A.D. Martin, W.J. Stirling and R.G. Roberts, Phys. Lett. B306 (1993) 145 .

[7] K.J. Eskola, K. Kajantie and P.V. Ruuskanen, Phys. Lett. B332 (1994) 191.

[8] K.J. Eskola, Nucl. Phys. A590 (1995) 383c.

[9] A.D. Martin, W.J. Stirling and R.G. Roberts, RAL-93-077, J. Phys. G19 (1993) 1429.

[10] D.W. Duke and J.F. Owens, Phys. Rev. D30 (1984) 49.

[11] A.D. Martin, W.J. Stirling and R.G. Roberts, Phys. Rev. D50 (1994) 6734 .

[12] H. Satz, Nucl. Phys. A544 (1992) 371c.

[13] T. Matsui and H. Satz, Phys. Lett. B178 (1986) 416.

[14] F. Karsch, M.T. Mehr, and H. Satz, Z. Phys. C37 (1988) 617.

[15] S. Ramos et al., NA38 Collab., Nucl. Phys. A590 (1995) 117c and references therein.

[16] M. Gonin et al. in proceedings of Quark Matter '96, Heidelberg, Germany, P. Braun-Munzinger et al., eds.

[17] S. Gavin and R. Vogt, Nucl. Phys. B345 (1990) 104.

[18] S. Gavin, H. Satz, R.L. Thews, and R. Vogt, Z. Phys. C61 (1994) 351.

[19] D. Kharzeev and H. Satz, CERN preprint, CERN-TH-95-214, August 1995. 
[20] S. Gavin and R. Vogt, hep-ph/9606490; S. Gavin and R. Vogt in proceedings of Quark Matter '96, Heidelberg, Germany, P. Braun-Munzinger et al., eds.

[21] CMS Technical Proposal, CERN/LHCC 94-38, LHCC/P1 (1994).

[22] F. Karsch and H. Satz, Z. Phys. C51 (1991) 209.

[23] B. Petersson, Nucl. Phys. A525 (1991) 237c.

[24] D.J. Gross, R.D. Pisarski, and L.G. Yaffe, Rev. Mod. Phys. 53 (1981) 43.

[25] For a recent review, see G.A. Schuler, CERN Preprint CERNTH.7170/94, February 1994 and references therein.

[26] M.L. Mangano, P. Nason and G. Ridolfi, Nucl. Phys. B405 (1993) 507.

[27] R.V. Gavai et al., Int. J. Mod. Phys. A10 (1995) 3043.

[28] G.A. Schuler and R. Vogt, CERN-TH/96-157 and LBL-39012, Phys. Lett. $\mathbf{B}$ in press.

[29] CDF Collaboration, Fermilab preprint, Fermilab-Conf-94/136-E, May 1994.

[30] L. Antoniazzi et al., Phys. Rev. Lett. 70 (1993) 383.

[31] L. Antoniazzi et al., Phys. Rev. D46 (1992) 4828.

[32] B. Ronceux, NA38 Collab., Nucl. Phys. A566 (1994) 371c.

[33] K. Ueno et al., Phys. Rev. Lett. 42 (1979) 486.

[34] T. Yoshida et al., Phys. Rev. D39 (1989) 3516.

[35] G. Moreno et al., Phys. Rev. D43 (1991) 2815.

[36] F. Abe et al., Fermilab preprint, Fermilab-Pub-95/271-E, August 1995.

[37] S.J. Brodsky, C. Peterson and N. Sakai, Phys. Rev. D23 (1981) 2745;

S.J. Brodsky, P. Hoyer, C. Peterson and N. Sakai, Phys. Lett. 93B (1980) 451.

[38] J. Badier et al., Phys. Lett. 86B (1979) 98; S. Childress et al., Phys. Rev. Lett. 55 (1985) 1962. 
[39] D. Antreasyan et al., Phys. Rev. Lett. 45 (1980) 863; C. Kourkoumelis et al., Phys. Lett. 91B (1980) 481; A.L.S. Angelis et al., Phys. Lett. 87B (1979) 398.

[40] M. Glück, E. Reya and A. Vogt, Z. Phys. C53 (1992) 127.

[41] C. Albajar et al., Phys. Lett. B186 (1987) 237; K. Eggert and A. Morsch (UA1), private communication in [27].

[42] N.S. Craigie, Phys. Rep. 47 (1978) 1.

[43] R. Vogt, Atomic Data and Nuclear Data Tables 50 (1992) 343.

[44] Particle Data Group, K. Hikasa et al., Phys. Rev. D45 (1992) S1.

[45] R. Baier and R. Rückl, Z. Phys. C19 (1983) 251.

[46] P. Cho and A.K. Leibovich, Caltech Preprint, CALT-68-1988, May 1995, hep-ph/9505329; Caltech Preprint, CALT-68-2026, November 1995, hep-ph/9511315.

[47] E. Braaten, M.A. Doncheski, S. Fleming and M.L. Mangano, Phys. Lett. B333 (1994) 548.

[48] D.M. Alde et al., Phys. Rev. Lett. 66 (1991) 2285.

[49] K.J. Eskola, Nucl. Phys. B400 (1993) 240.

[50] M.E. Peskin, Nucl. Phys. B156 (1979) 365.

[51] G. Bhanot and M.E. Peskin, Nucl. Phys. B156 (1979) 391.

[52] D. Kharzeev, H. Satz, A. Syamtomov and G. Zinovjev, CERN preprint CERN-TH/96-72, hep-ph/9605448.

[53] G. Zinovjev, private communication.

[54] J. Hüfner and B. Povh, Phys. Rev. Lett. 58 (1987) 1612.

[55] R. Vogt, S.J. Brodsky and P. Hoyer, Nucl. Phys. B360 (1991) 97.

[56] S. Liuti and R. Vogt, Phys. Rev. C51 (1995) 2244.

[57] M. Arneodo, Phys. Rep. 240 (1994) 301.

[58] S. Gavin and M. Gyulassy, Phys. Lett. B214 (1988) 241.

[59] J.D. Bjorken, Phys. Rev. D27 (1983) 140. 
[60] M.-C. Chu and T. Matsui, Phys. Rev. D37 (1988) 1851.

[61] J.-P. Blaizot and J.-Y. Ollitrault, Phys. Lett. 199B (1987) 499.

[62] F. Karsch and R. Petronizio, Phys. Lett. 212B (1988) 255.

[63] J.I. Kapusta, L. McLerran and D.K. Srivastava, Phys. Lett. B283 (1992) 145.

[64] R. Vogt, B.V. Jacak, P.L. McGaughey and P.V. Ruuskanen, Phys. Rev. D49 (1994) 3345.

[65] X.-M. Xu, D. Kharzeev, H. Satz and X.-N. Wang, Phys. Rev. C53 (1996) 3051.

[66] X.-N. Wang and M. Gyulassy, Phys. Rev. D44 (1991) 3501; Comp. Phys. Commun. 83 (1993) 133.

[67] J. Collins, D. Soper and G. Sterman, Nucl. Phys. B250 (1985) 199; J. Collins and D. Soper, Nucl. Phys. B193 (1981) 381; B213 (1983) 545(E); B197 (1982) 446.

[68] C. Davies, B. Webber and W.J. Stirling, Nucl. Phys. B256 (1985) 413.

[69] G. Altarelli, R.K. Ellis, M. Greco and G. Martinelli, Nucl. Phys. B246 (1984) 12.

[70] P.B. Arnold and R. Kauffman, Nucl. Phys. B349 (1991) 381.

[71] G.A. Ladinsky and C.-P. Yuan, MSUHEP-93/20.

[72] M.H. Reno, UIOWA-94-01.

[73] The PHENIX Conceptual Design Report, 1993 (unpublished); PHENIX/Spin Collaboration Report, 1995 (unpublished).

[74] Z. Lin and M. Gyulassy, Phys. Rev. Lett. 77 (1996) 1222.

[75] S. Gavin, P.L. McGaughey, P.V. Ruuskanen and R. Vogt, hepph/9604369 (1996), Phys. Rev. C, in press.

[76] BRAHMS Conceptual Design Report, 1994 (updated 1995); F. Videbaek, BRAHMS Collaboration, Nucl. Phys. A566 (1994) 299c.

[77] J. Ftáčnik, P. Lichard and J. Pišút, Phys. Lett. B207 (1988) 194.

[78] S. Gavin, M. Gyulassy and A. Jackson, Phys. Lett. B207 (1988) 257. 
[79] R. Vogt, M. Prakash, P. Koch and T.H. Hansson, Phys. Lett. B207 (1988) 263.

[80] ATLAS Technical Proposal, CERN/LHCC/94-43, LHCC/P2 (1994).

[81] S.J. Brodsky, A. Hebecker, and E. Quack, hep-ph/9609384.

[82] B. Kopeliovich and B. Povh, nucl-th/9607035.

[83] Contributions to 'Light and Heavy Nuclei in HERA' subgroup (convenors: M. Arneodo, A. Bialas, M. Krasny, T. Sloan, G. van der Steenhoven, M. Strikman) in Proceedings of Future Physics at HERA, eds. G. Ingelman, A. De Roeck, R. Klanner, DESY report.

[84] These statements are based on the calculations of Ref. [75] for the LHC (using the acceptances of the ALICE experiment). 